\title{
Neural fields with rebound currents: novel routes to patterning *
}

\author{
Sunil Modhara ${ }^{\dagger}$, Yi Ming Lai ${ }^{\ddagger}$, Rüdiger Thul§, and Stephen Coombes $₫$
}

\begin{abstract}
The understanding of how spatio-temporal patterns of neural activity may arise in the cortex of the brain has advanced with the development and analysis of neural field models. To replicate this success for sub-cortical tissues, such as the thalamus, requires an extension to include relevant ionic currents that can further shape firing response. Here we advocate for one such approach that can accommodate slow currents. By way of illustration we focus on incorporating a T-type calcium current into the standard neural field framework. Direct numerical simulations are used to show that the resulting tissue model has many of the properties seen in more biophysically detailed model studies, and most importantly the generation of oscillations, waves, and patterns that arise from rebound firing. To explore the emergence of such solutions we focus on one- and two-dimensional spatial models and show that exact solutions describing homogeneous oscillations can be constructed in the limit that the firing rate nonlinearity is a Heaviside function. A linear stability analysis, using techniques from non-smooth dynamical systems, is used to determine the points at which bifurcations from synchrony can occur. Furthermore, we construct periodic travelling waves and investigate their stability with the use of an appropriate Evans function. The stable branches of the dispersion curve for periodic travelling waves are found to be in excellent agreement with simulations initiated from an unstable branch of the synchronous solution.
\end{abstract}

Key words. Neural field, pattern formation, non-smooth dynamical systems, synchrony, periodic travelling waves, Evans function.

AMS subject classifications. 92C20, 92B20, 39B82, 35C07.

1. Introduction. There are a zoo of ionic currents that can shape the firing response of a single neuron seen in electrophysiological studies, and in particular patch-clamp recordings [16]. From a modelling perspective these currents are commonly described using extensions of the Hodgkin-Huxley model to include further gating variables for the opening and closing of ion channels [13]. The high dimensionality of the resulting point model favours analysis using perturbation methods, such as geometric singular perturbation theory $[18,30]$, and is often complemented by numerical bifurcation analysis [24]. This approach does not extend well to treating very large networks of synaptically coupled neurons relevant to understanding large scale spatio-temporal rhythms seen in brain tissue. Here, mean field type reductions are often favoured that ignore or wash out the detailed ionic mechanisms that can sculpt firing patterns. This is the basis for many continuum neural field models of cortex where it is typically assumed that the firing rate of a population of neurons is a sigmoidal function solely of synaptic activity,

\footnotetext{
*Submitted to the editors .

${ }^{\dagger}$ Centre for Mathematical Medicine and Biology, School of Mathematical Sciences, University of Nottingham, University Park, Nottingham, NG7 2RD, UK (sunil.modhara@nottingham.ac.uk).

${ }^{\ddagger}$ School of Medicine, University of Nottingham, Queen’s Medical Centre, Nottingham, NG7 2UH, UK (pmzyl@exmail.nottingham.ac.uk).

${ }^{\S}$ Centre for Mathematical Medicine and Biology, School of Mathematical Sciences, University of Nottingham, University Park, Nottingham, NG7 2RD, UK (ruediger.thul@nottingham.ac.uk).

"Centre for Mathematical Medicine and Biology, School of Mathematical Sciences, University of Nottingham, University Park, Nottingham, NG7 2RD, UK (stephen.coombes@nottingham.ac.uk).
} 
and see $[7,4]$ for an overview of this approach. When this sigmoid is taken sufficiently steep so as to be replaced by a Heaviside function, then a plethora of mathematical results for localised patterns and waves can be generated, as illustrated in [1, 23, 8, 11]. However, by ignoring any possible dependence of firing rate mechanisms on intrinsic ionic currents the standard neural field approach cannot be expected to reproduce all biological firing behaviours. A case in point is so-called rebound firing, whereby neuronal response to release of hyperpolarising inhibition can result in a burst of action potentials [10]. An example of this behaviour can be found in thalamo-cortical relay cells that possess T-type $\mathrm{Ca}^{2+}$ channels. When $\mathrm{Ca}^{2+}$ enters the neuron through these channels a large voltage depolarisation known as the low-threshold $\mathrm{Ca}^{2+}$ spike (LTS) can occur. Conventional action potentials mediated by fast $\mathrm{Na}^{+}$and $\mathrm{K}^{+}$currents can ride on the crest of an LTS resulting in a burst response (i.e., a tight cluster of several voltage spikes). A minimal model of this process comes in the form of a four dimensional ordinary differential equation model developed by Wang [29], and its bursting properties have been dissected using a fast-slow analysis by Guckenheimer et al. [12]. Numerical simulations of this type of spiking model in spatially structured networks with interactions mediated entirely by inhibitory synapses demonstrate the possibility of wave progagation [25]. After some model reduction (that essentially tracks the LTS, though not the voltage spikes in a burst) these waves can be analysed, at least to some extent, using singular perturbation methods [31]. However, this approach does not easily allow for the determination of solution stability or bifurcation. Thus, it is of interest to look for strategies that allow for the successes of the neural field approach to incorporate some of the important biology from single cell modelling, so as to better understand the dynamics of sub-cortical structures, and in particular the thalamus. This is especially important given that this organ is the sensory gateway to the cortex [26], and is famed for its role in the generation of thalamo-cortical rhythms [28].

The approach we advocate for here augments the standard neural field approach with the use of a firing rate that is a function of some underlying voltage model. This voltage model does not attempt to describe spikes per se, merely the envelopes upon which they ride, as is the case for the LTS. This approach has previously been developed in [5] for capturing the dynamics of an integrate-and-fire model with a slow T-type $\mathrm{Ca}^{2+}$ current [27], yet generalises to include other slow ionic currents [6]. The use of the voltage model means that the neural field can include a representation of the gating variables for intrinsic (nonspiking) ionic currents. Moreover, in the Heaviside limit many of the mathematical techniques for treating standard neural fields can be utilised albeit with one caveat. This being that the gating variables be described by a piecewise-linear or piecewise constant nonlinear dynamical system. Although a seemingly stringent choice this is reasonable when recognising that many of the sigmoidal activation/inactivation curves for gating variables can be approximated this way. The simplest choice is to adopt a switch-like perspective, and this is what we pursue here by considering activation/in-activation curves to be Heaviside functions. However, this means that the dynamics is non-smooth, and one must be careful not to abuse methodologies that are valid only for smooth systems. We avoid this potential pitfall by making extensive use of saltation operators when treating the stability of network solutions. Crucially, we show how the standard derivation of saltation operators, which was originally performed for ordinary differential equations [22], can be extended to capture non-local interactions as used in the present study. We focus on spatially continuous purely inhibitory neural field models that 


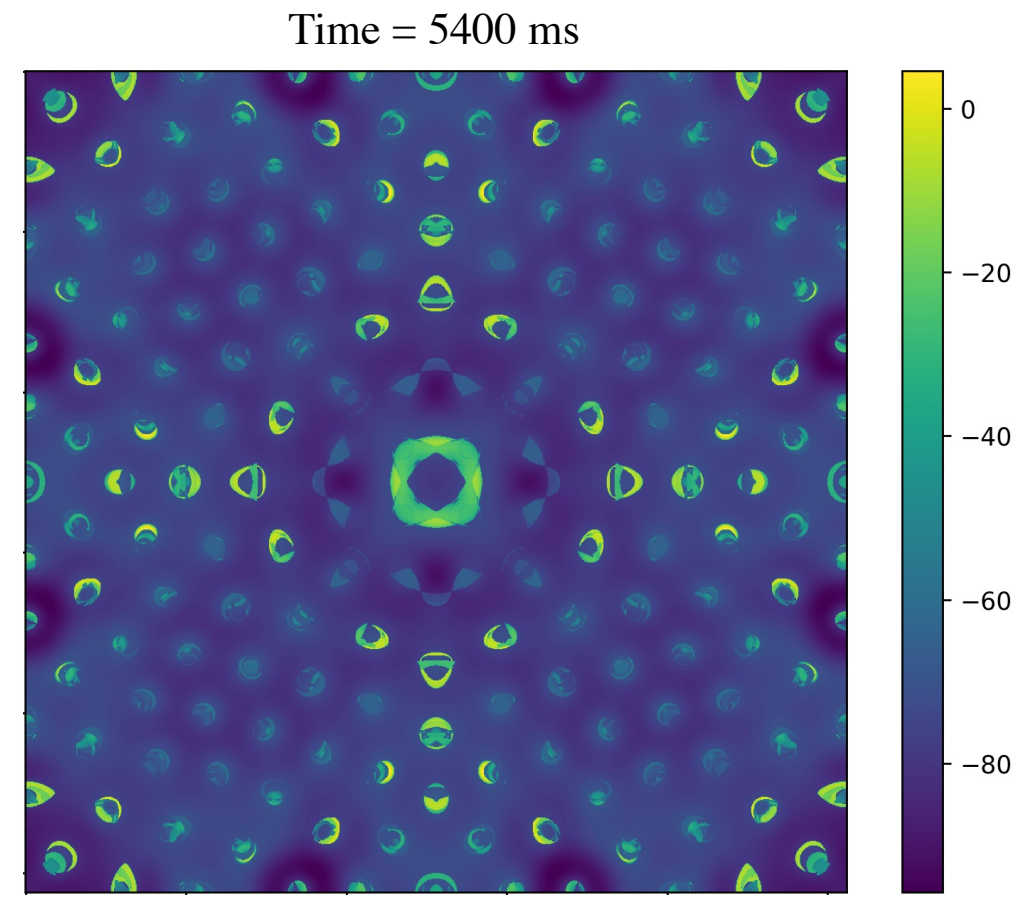

Figure 1: Membrane voltage $v$ in $\mathrm{mV}$ on a two-dimensional domain of size $0.38 \times 0.38 \mathrm{~cm}$ with periodic boundary conditions shown at a fixed time point. Simulations were performed with $1024 \times 1024$ spatial grid points. Parameter values as in Table 1. See Additional movie $1 a$ and the higher temporal resolution Additional movie $1 b$ in the Supplementary Material for further illustration.

support patterned states via rebound. The inclusion of rebound currents can lead to complex spatio-temporal patterns as illustrated in Figure 1 and Additional movies $1 a$ and $1 b$ in the Supplementary Material. Starting from a bump of elevated synaptic activity in the centre of the domain, patterns emerge where spatially separated and distinct parts of the domain fire in synchrony. Intriguingly, the patterns generated by the $I_{\mathrm{T}}$ current here are qualitatively similar to those in [3], which originate from an $I_{\mathrm{h}}$ current. This suggests that generic rebound currents may be capable of producing non-trivial patterns. While we focus on the existence and linear stability of synchronous oscillations and travelling waves, by way of example, we stress that the approach presented here is more broadly applicable.

In section 2, we introduce a generalised continuum neural field model and describe how it can incorporate a slow T-type calcium current. To illustrate the pattern forming properties of the model we show numerical simulations for a planar realisation of a purely inhibitory network. For sufficiently slow synaptic interactions we see synchronised activity and the formation of spatially structured travelling waves. In contrast to patterns seen in standard neural field models with short range excitation and long range inhibition, these do not arise through a Turing instability and instead depend heavily upon a rebound mechanism. We consider the construction and stability of the synchronous solution in section 3, making use of 
tools from non-smooth dynamical systems. This is used to make predictions about parameter regimes for the destabilisation of homogeneous oscillations that can give way to inhomogeneous patterned states. Next, in section 4 we turn our attention to periodic travelling waves in one spatial dimension and the construction of dispersion curves (speed vs. period). Wave stability is determined with the calculation of an appropriate Evans function in section 5, and direct numerical simulation suggests that unstable waves lie in the basin of attraction of stable periodic travelling waves. Finally, in section 6 we discuss natural extensions to the work in this paper.

2. The model. Neural field modelling has been a mainstay for helping to understand cortical activity since its introduction in the 1970s, and see [7] for a recent review of the theory and its many applications. In this continuum approach non-local spatial interactions are mediated by a connectivity kernel that allows firing rate activity to be transferred from one point in the tissue model to another. In their simplest form they are often written as integro-differential equations of the type

$$
Q u=\psi, \quad \psi=w \otimes f
$$

Here, $u$ represents the level of synaptic activity and $Q$ is a temporal differential operator that describes synaptic processing. The source term $\psi$ is non-local, and is formed from the spatial convolution $(\otimes)$ of an anatomically motivated kernel function $w$ and the nonlinear firing rate function $f$. The equations of motion (2.1) are typically closed by making the firing rate a (typically sigmoidal) function of $u$ so that $f=f(u)$. Although this has proven remarkably useful for modelling cortical tissue it cannot hope to model sub-cortical structures like the thalamus where intrinsic nonlinear ionic currents can dominate the firing rate response [9] . A minimal extension to cortical neural field modelling has been proposed in [5] to accommodate slow ionic currents. In this approach the equations of motion are closed in a way that couples to the intrinsic gating variables of the relevant ionic currents, which in our case is the slow T-type calcium current $I_{\mathrm{T}}$. This is achieved with the inclusion of a new variable that tracks the voltage envelope $v$ upon which a burst of spikes can ride and closing the equations with the choice $f=f(v)$. The dynamics for $v$ preserves all the slow currents that would drive a single neuronal cell and drops those currents responsible for the detailed shape of the action potential (fast sodium and potassium currents). This approach has been shown to capture the qualitative burst and tonic response properties of thalamic networks built from spiking reticular and thalamo-cortical relay cells [15]. Given the mathematical simplicity of this modelling approach over other choices, such as biophysical cell based networks [25], this is the one we adopt here.

In more detail consider a continuum description of thalamo-cortical relay cells defined on the infinite plane and introduce a voltage envelope variable $v=v(\mathbf{r}, t), \mathbf{r} \in \mathbb{R}^{2}, t>0$, with dynamics

$$
C \frac{\partial}{\partial t} v=I_{\mathrm{L}}+I_{\mathrm{T}}+I_{\mathrm{syn}} .
$$

The left hand side of the current balance equation (2.2) is the capacitative current through a patch of neuronal membrane with capacitance $C$, whilst the right hand side describes the 
three main (non-spiking) currents for leak $\left(I_{\mathrm{L}}\right)$, T-type $\mathrm{Ca}^{2+}$ channels $\left(I_{\mathrm{T}}\right)$, and synaptic input, respectively $\left(I_{\mathrm{syn}}\right)$. The leak current has a simple ohmic form $I_{\mathrm{L}}=g_{\mathrm{L}}\left(v_{\mathrm{L}}-v\right)$ for some constant leak conductance $g_{\mathrm{L}}$ and leak reversal potential $v_{\mathrm{L}}$, whilst the dynamics for $I_{\mathrm{T}}$ is governed by a gating variable $h$ with $I_{\mathrm{T}}=g_{\mathrm{T}} h m_{\infty}(v)\left(v_{\mathrm{T}}-v\right)$, with $g_{\mathrm{T}}$ and $v_{\mathrm{T}}$ representing constant conductance and reversal potential, respectively. The activation function $m_{\infty}(v)$, describing a fast switching process with respect to a voltage threshold at $v=v_{\mathrm{h}}<v_{\mathrm{L}}$ (negative with respect to rest), is given simply by $m_{\infty}(v)=H\left(v-v_{\mathrm{h}}\right)$, where $H$ is a Heaviside step function. The slower inactivation dynamics for $h$ is governed by

$$
\frac{\partial}{\partial t} h=\frac{h_{\infty}(v)-h}{\tau_{\mathrm{h}}(v)} .
$$

Here, $h_{\infty}(v)=H\left(v_{\mathrm{h}}-v\right)$ and $\tau_{\mathrm{h}}(v)=\tau_{\mathrm{h}}^{-} H\left(v-v_{\mathrm{h}}\right)+\tau_{\mathrm{h}}^{+} H\left(v_{\mathrm{h}}-v\right)$ so that for $v>v_{\mathrm{h}}, h$ decays to zero at a rate $\tau_{\mathrm{h}}^{-}$and otherwise grows exponentially to one at a rate $\tau_{\mathrm{h}}^{+}$. Thus, if the voltage $v$ is ever hyperpolarised below $v_{\mathrm{h}}$ by a transient inhibitory synaptic current then $h$ will increase though its effect will not be felt in the $I_{\mathrm{T}}$ current until inhibition wears off and $v$ increases back to rest by crossing through $v_{\mathrm{h}}$ from below. Since $v_{\mathrm{T}}$ is large and positive with respect to rest there is a jump in $I_{\mathrm{T}}$, which will then decay while $v$ remains above $v_{\mathrm{h}}$, hence why it is referred to as a rebound current. The positive rebound current drives voltage activity up and can lead to an increase or a burst in firing. This is modelled with the simple choice $f=f(v)$. In common with standard firing rate models we could take this to be a sigmoid, though for reasons of mathematical tractability we shall make the idealised choice

$$
f(v)=\frac{1}{\tau_{\mathrm{R}}} H\left(v-v_{\mathrm{th}}\right) .
$$

Here $v_{\text {th }}$, with $v_{\mathrm{h}}<v_{\mathrm{L}}<v_{\mathrm{th}}$, is a firing threshold such that above this the tissue fires at a rate limited by the refractory time-scale $\tau_{\mathrm{R}}$ and otherwise is quiescent. The model is completed with the choice of synaptic dynamics by setting $I_{\mathrm{syn}}=g_{\mathrm{syn}} u$, with $u$ as in (2.1). We stress here that $g_{\mathrm{syn}}$ is not a conductance per se, but is rather a signed, strength of interaction. We shall focus on a tissue model with inhibitory connections and an off-centre pattern of connectivity which has previously been shown to favour smoothly propagating waves in thalamic networks $[25,31]$. The typical length scale for inhibitory connections in thalamic networks is of the order of $0.2 \mathrm{~mm}[25]$. We do this by setting

$$
\psi(\mathbf{r}, t)=\int_{\Gamma} w\left(\left|\mathbf{r}-\mathbf{r}^{\prime}\right|\right) f \circ v\left(\mathbf{r}^{\prime}, t\right) \mathrm{d} \mathbf{r}^{\prime}
$$

with $w(r)=w_{0} \Omega(r)$ and

$$
\Omega(r)=\Omega_{0} \exp (-r / \sigma)(1-\gamma \cos (\rho r / \sigma)), \quad 0<\gamma \leq 1 .
$$

We set $w_{0}=-1$ throughout this study and choose $\Omega_{0}$ in such a way that the kernel $\Omega(r)$ is normalised, i.e. $\int_{\Gamma} \Omega(|\mathbf{r}|) \mathrm{d} \mathbf{r}=1$. This results in $\Omega_{0}=\left(\rho^{2}+1\right) /\left(2 \sigma\left(\rho^{2}-\gamma+1\right)\right)$ for $\Gamma=\mathbb{R}$ and $\Omega_{0}=\left(\rho^{2}+1\right)^{2} /\left(2 \pi \sigma^{2}\left(\rho^{4}+(\gamma+2) \rho^{2}-\gamma+1\right)\right)$ for $\Gamma=\mathbb{R}^{2}$. The length scale $\sigma$ measures the spatial decay of thalamic connections, and $\gamma$ and $\rho$ determine the off-centre and oscillatory 
Figure 2: Illustration of the radially symmetric off-center connectivity kernel $\Omega(r)$ from (2.6) in $2 \mathrm{D}$.

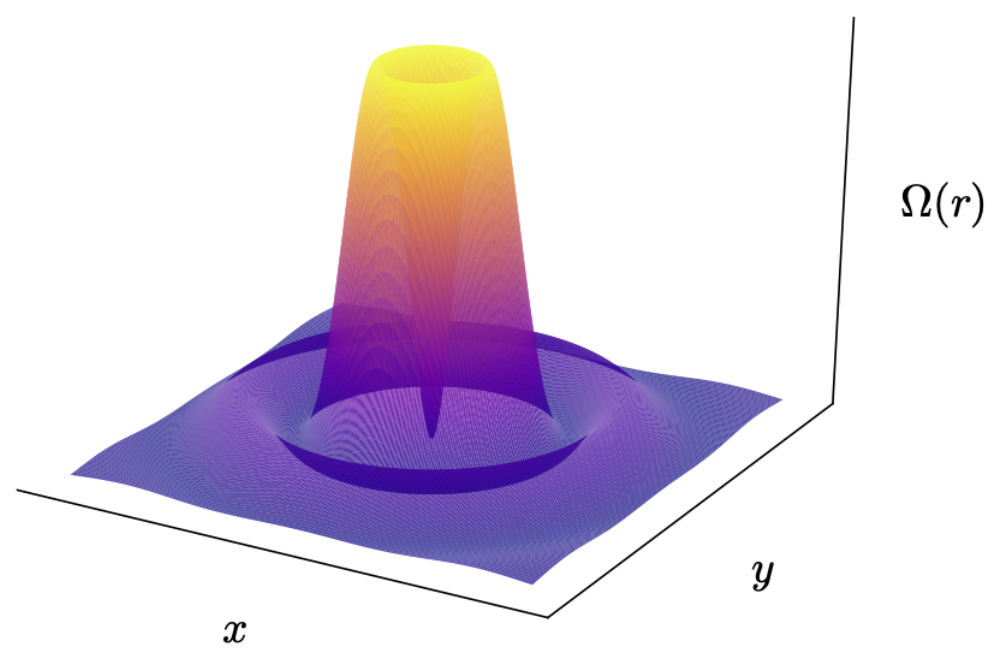

nature of the kernel, respectively. See Figure 2 for an illustration of the off-centre kernel shape. The finite rise and fall time of a post synaptic response is modelled using a second order differential operator:

$$
Q=\left(1+\frac{1}{\alpha} \frac{\partial}{\partial t}\right)^{2},
$$

where $\alpha^{-1}$ is the time-to-peak. The Green's function of the linear differential operator (2.7) is an $\alpha$-function given by $\eta(t)=\alpha^{2} t \mathrm{e}^{-\alpha t} H(t)$. Hence, we can also write the model (2.1) in the integral form

$$
u(\mathbf{r}, t)=\int_{0}^{\infty} \eta(s) \psi(\mathbf{r}, t-s) \mathrm{d} s .
$$

Given the large value of $v_{\mathrm{T}}$ (of around $150 \mathrm{mV}$ with respect to rest) it is practical to make the approximation $v_{\mathrm{T}}-v \simeq v_{\mathrm{T}}$ and absorb this factor with $g_{\mathrm{T}}$ to obtain the reduction $I_{\mathrm{T}}=g_{\mathrm{T}} h H\left(v-v_{\mathrm{h}}\right)$. Note that $g_{\mathrm{T}}$ is no longer a conductance as such, but is a scaled, signed strength of interaction, with units $\mathrm{mV} \mathrm{mS} / \mathrm{cm}^{2}$. We note that in the original formulation described in [5] that a simplification was made whereby $v$ was replaced by its quasi-steady state value (obtained by setting the right hand side of (2.2) to zero). Here, we shall lift this restriction (which would require the membrane time-scale $C / g_{\mathrm{L}}$ to be much shorter than $\tau_{\mathrm{h}}^{ \pm}$ and $\alpha^{-1}$, which is often not the case in neurobiology). For the following, it is convenient to rewrite the second-order equation (2.1) as two first order equations by introducing the new variable

$$
r(\mathbf{r}, t)=\left(1+\frac{1}{\alpha} \frac{\partial}{\partial t}\right) u(\mathbf{r}, t) .
$$



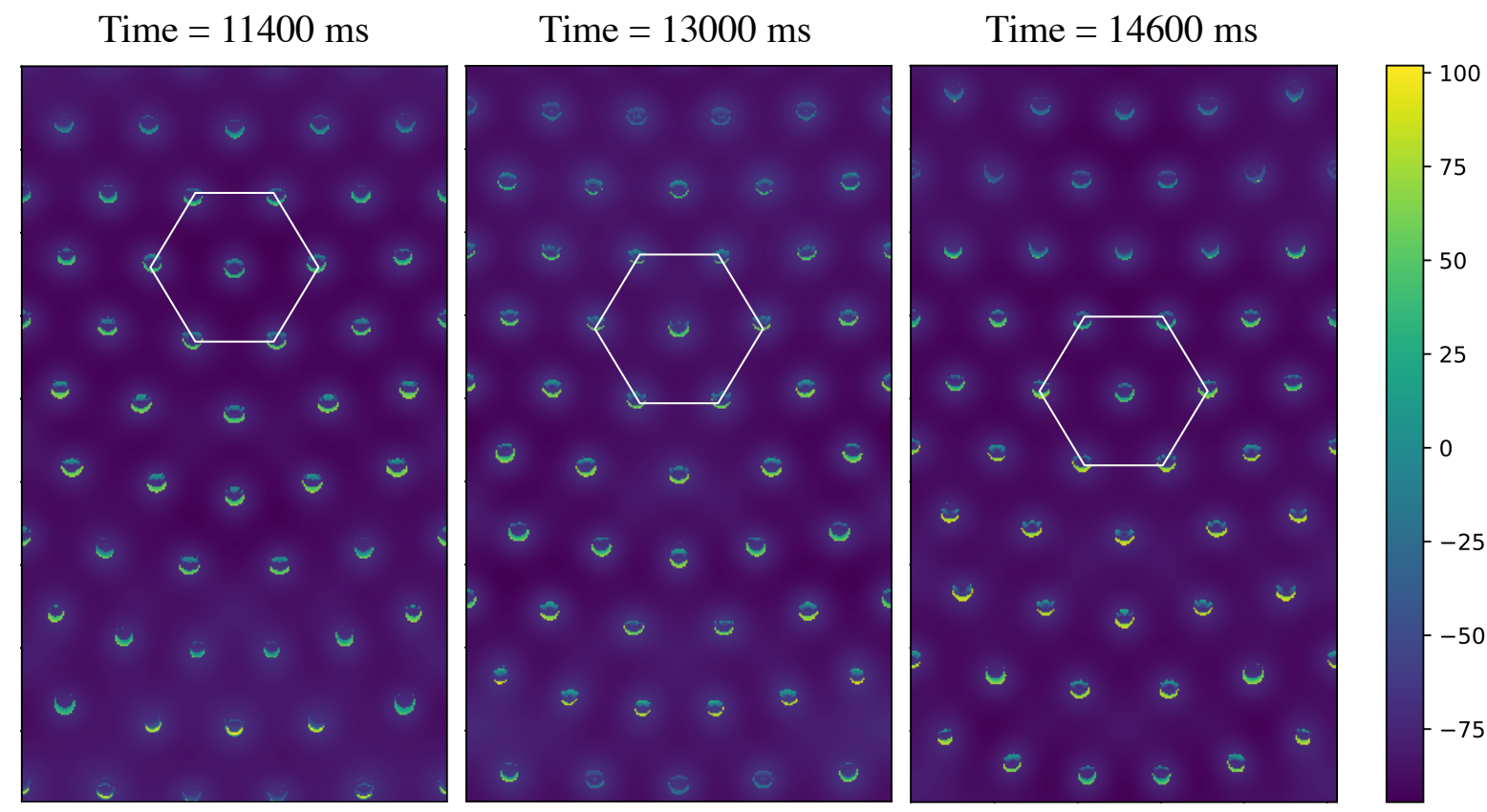

Figure 3: Membrane voltage $v$ in $\mathrm{mV}$ on a two-dimensional domain of size $0.16 \times 0.27 \mathrm{~cm}$ with periodic boundary condition for three different time points. The white hexagon connects the same six spots of large membrane depolarisation, illustrating a moving hexagonal pattern. Simulations were performed with $512 \times 886$ spatial grid points. Parameter values as in Table 1 and $\alpha=0.19$ and $g_{T}=20.3 \mathrm{mVmS} / \mathrm{cm}^{2}$. See Additional movies $2 a$ and $2 b$ in the Supplementary Material for further illustration.

In addition to complex spatio-temporal patterns as illustrated in Figure 1, our model also supports travelling wave solutions. An example for this is depicted in Figure 3. It shows a wave of rigidly moving hexagons, as can be gleaned from the vertical translation of the white hexagon, which connects the same six spots of large membrane depolarisation. We refer the reader to Additional movie $2 a$ in the Supplementary Material for further illustration and Additional movie $2 b$ showing the translation of a unit hexagonal cell down the domain. While the travelling wave in Figure 3 emerges from a hexagonally patterned initial condition, we also found periodic travelling waves in $2 \mathrm{D}$ upon perturbation of a synchronous network state. These patterns do not arise via a Turing mechanism from a spatially homogeneous steady state. To understand this phenomenon, we will next construct the synchronous network state and determine its linear stability. For mathematical tractability, we will present our analysis in one spatial dimension, and will not further investigate $2 \mathrm{D}$ patterns. To ease the presentation, it is convenient to collect the four state variables into a vector $z=z(x, t)=(v, u, r, h)$ with $x \in \mathbb{R}$. For completeness, we here recapitulate the equations for the spatially extended non- 


\begin{tabular}{c|c|l}
\hline Parameter & Value & Description \\
\hline \hline$g_{\mathrm{L}}$ & $0.035 \mathrm{mS} / \mathrm{cm}^{2}$ & Overall leak current conductance strengh \\
$v_{L}$ & $-65.0 \mathrm{mV}$ & Leak current reversal potential \\
$g_{\mathrm{T}}$ & $8.4 \mathrm{mVmS} / \mathrm{cm}^{2}$ & Scaled strength of $I_{T}$ interaction \\
$\tau_{+}$ & $100.0 \mathrm{~ms}$ & $I_{T}$ saturation time constant \\
$\tau_{-}$ & $20.0 \mathrm{~ms}$ & $I_{T}$ decay time constant \\
$v_{\mathrm{th}}$ & $-35.0 \mathrm{mV}$ & Firing threshold \\
$v_{\mathrm{h}}$ & $-70.0 \mathrm{mV}$ & Rebound threshold \\
\hline$\alpha$ & $0.1 \mathrm{~ms}^{-1}$ & Synaptic time constant \\
$C$ & $1.0 \mu \mathrm{F} / \mathrm{cm}^{2}$ & Membrane capacitance \\
$\tau_{R}$ & $5.0 \mathrm{~ms}$ & Firing strength scaling constant \\
$g_{\mathrm{syn}}$ & $200.0 \mathrm{mVmS} / \mathrm{cm}^{2}$ & Scaled strength of synaptic interaction \\
$\sigma$ & $0.02 \mathrm{~cm}$ & Length scale of spatial connectivity \\
$\gamma$ & 1.0 & Measure of off-centre connectivity \\
$\rho$ & 2.0 & Measure of oscillatory nature in connectivity
\end{tabular}

Table 1: Standard parameter values. The upper half contains parameter values obtained from fits with experimental data [27]. The remaining parameter values are specific to the present study. Note that $g_{\mathrm{T}}$ and $g_{\mathrm{syn}}$ are compound parameters measuring the strength of currents. See text for details.

local model:

$$
\begin{aligned}
C \frac{\partial}{\partial t} v & =I_{\mathrm{L}}+I_{\mathrm{T}}+I_{\mathrm{syn}}, \\
\frac{\partial}{\partial t} u & =\alpha(r-u), \\
\frac{\partial}{\partial t} r & =\alpha(w \otimes f(v)-r), \\
\frac{\partial}{\partial t} h & =\frac{h_{\infty}(v)-h}{\tau_{\mathrm{h}}(v)},
\end{aligned}
$$

where we used the spatial convolution operator $\otimes$ introduced in (2.1). Parameter values are provided in Table 1.

3. The synchronous solution. Neural tissue often exhibits synchronous behaviour, and this is especially true within the circuitry of the thalamus with a robust post-inhibitory rebound mechanism for the firing of thalamo-cortical relay neurons [17]. We therefore begin our analysis by constructing the synchronous solution $z(x, t)=z(t)$ for all $x$. We find from (2.10) 
that the dynamics of $z(t)$ is governed by

$$
\begin{aligned}
\frac{\mathrm{d}}{\mathrm{d} t} v & =g_{\mathrm{L}}\left(v_{\mathrm{L}}-v\right)+g_{\mathrm{T}} h H\left(v-v_{\mathrm{h}}\right)+g_{\mathrm{syn}} u, \\
\frac{\mathrm{d}}{\mathrm{d} t} u & =\alpha(r-u), \\
\frac{\mathrm{d}}{\mathrm{d} t} r & =\alpha\left(w_{0} f(v)-r\right), \\
\frac{\mathrm{d}}{\mathrm{d} t} h & =\frac{h_{\infty}(v)-h}{\tau_{\mathrm{h}}(v)} .
\end{aligned}
$$

Here, we used the fact that for the synchronous state, the argument of $f$ in (2.10c) does not depend on space and $w$ is normalised to $w_{0}=-1$. For later, it is convenient to express (3.1) as $\mathrm{d} z / \mathrm{d} t=F(z)$. Since $f, h_{\infty}$, and $\tau_{h}$ are piecewise constant functions, the system (3.1) is piecewise linear with switching manifolds at $v=v_{\mathrm{h}}$ and $v=v_{\text {th }}$. We can therefore divide the phase space into three regions: (i) $v<v_{\mathrm{h}}$, (ii) $v_{\mathrm{h}}<v<v_{\text {th }}$ and (iii) $v>v_{\text {th }}$. In each of these regions and hence between switching events, (3.1) can be solved explicitly. This is particularly straightforward since (3.1c) and (3.1d) decouple, and the solutions for $r(t)$ and $h(t)$ can be used as time-dependent input for (3.1a) and (3.1b), respectively. Figure 4 shows the resultant synchronous orbit in the $(v, h)$ plane. It is characterised by the timesof-flight $\Delta_{i}, i=1, \ldots, 4$, along the four distinct segments of the solution and an initial state $z(0)=\left(v_{\mathrm{h}}, u_{0}, r_{0}, h_{0}\right)$. The a priori seven unknowns - 4 times-of-flight and 3 unknown components of $z(0)$ - are determined by demanding continuity, periodicity and a sequence of switching events. In other words, solutions in consecutively visited regions are patched together in a continuous manner, and so to find the seven unknowns discussed above, we impose the seven conditions $v\left(T_{1}\right)=v_{\text {th }}, v\left(T_{2}\right)=v_{\text {th }}, v\left(T_{3}\right)=v_{\mathrm{h}}, v(T)=v_{\mathrm{h}}, u(0)=u(T)$, $r(0)=r(T)$, and $h(0)=h(T)$, where $T_{i}=\sum_{j=1}^{i} \Delta_{i}$ is the time of the $i$ th switching event and $T=T_{4}$ is the period of the synchronous solution.

Now that we have constructed the synchronous solution, we proceed by determining its linear stability. Consider a perturbation $\delta z(x, t)$ around the synchronous solution $z(t)$. Since the model equations change discontinuously at the switching manifolds, perturbations are mapped through the switching manifolds via saltation matrices $K_{i} \in \mathbb{R}^{4 \times 4}, i=1, \ldots, 4$, such that $\delta z\left(x, T_{i}^{+}\right)=K_{i} \delta z\left(x, T_{i}^{-}\right)$where $\delta z\left(x, T_{i}^{ \pm}\right)=\lim _{\epsilon \searrow 0} \delta z\left(x, T_{i} \pm \epsilon\right)$. Because of the nonlocal character of the model, we determine the components of $K_{i}$ via two separate approaches. For the rows of $K_{i}$ that pertain to $\delta v\left(x, T_{i}^{+}\right), \delta u\left(x, T_{i}^{+}\right)$and $\delta h\left(x, T_{i}^{+}\right)$, we employ standard approaches from non-smooth dynamical systems [22]. More precisely, the entries for $\delta v, \delta u$ and $\delta h$ correspond to the first, second and fourth row of

$$
K_{i}=I_{4}-\frac{\left(F_{i}^{-}-F_{i}^{+}\right)(\nabla g)^{T}}{(\nabla g) \cdot F_{i}^{-}},
$$

where $I_{n}$ denotes the identity matrix in $\mathbb{R}^{n \times n}$ and $F_{i}^{ \pm}=\lim _{\epsilon \searrow_{0}} F\left(z\left(T_{i} \pm \epsilon\right)\right)$ with $F$ defined as after (3.1). The function $g$ parameterises the switching manifolds and is either $g(z)=v-v_{\text {th }}$ or $g(z)=v-v_{\mathrm{h}}$, which results in $\nabla g=(1,0,0,0)^{T}$ in both cases. To populate the third row 


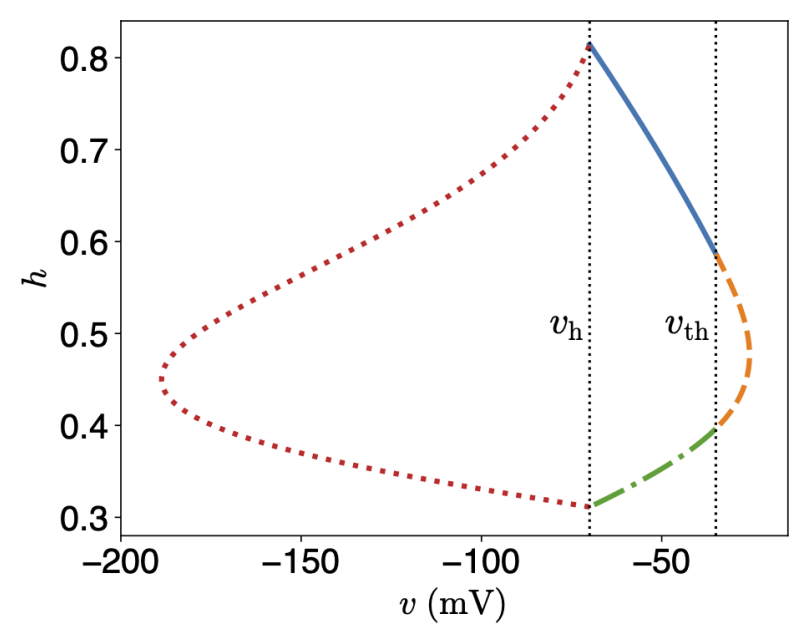

Figure 4: Synchronous period-1 orbit in the $(v, h)$ plane. Different colours (line styles) indicate parts of the orbit between switching events. The dotted lines represent the switching manifolds at $v_{\mathrm{h}}$ and $v_{\mathrm{th}}$, respectively. Parameter values as in Table 1 .

of $K_{i}$ and hence the entries that determine $\delta r\left(x, T_{i}^{+}\right)$, we start from

$$
\frac{\partial}{\partial t} r(x, t)=\alpha\left(-r(x, t)+\int_{-\infty}^{\infty} w\left(\left|x-x^{\prime}\right|\right) f \circ v\left(x^{\prime}, t\right) \mathrm{d} x^{\prime}\right) .
$$

Making the ansatz $\delta z(x, t)=\delta Z(t) \mathrm{e}^{i k x}$ and linearising around the synchronous state $z(t)$ results in

$$
\frac{\mathrm{d}}{\mathrm{d} t} \delta r(t)=\alpha\left(-\delta r(t)+\delta v(t) f^{\prime}(v(t)) \int_{-\infty}^{\infty} w\left(|x|^{\prime}\right) \mathrm{e}^{-i k x^{\prime}} \mathrm{d} x^{\prime}\right) .
$$

$$
\frac{\mathrm{d}}{\mathrm{d} t} \delta r(t)=\alpha\left(-\delta r(t)+\frac{\delta v(t) \widehat{w}(k)}{\tau_{R}} \sum_{i=1}^{2} \frac{\delta\left(t-T_{i}\right)}{\left|\dot{v}\left(T_{i}\right)\right|}\right),
$$

$$
\widehat{w}(k)=\int_{-\infty}^{\infty} w(|y|) \mathrm{e}^{-i k y} \mathrm{~d} y,
$$

represents the Fourier transform of $w(x)$ and the dot denotes differentiation with respect to synchronous voltage $v(t)$ crosses $v_{\text {th }}$ at times $T_{1}$ and $T_{2}$, respectively. Equation (3.5) shows that at the switching times $T_{1}$ and $T_{2}, \delta r(t)$ changes discontinuously according to

$$
\delta r\left(T_{i}^{+}\right)=\delta r\left(T_{i}^{-}\right)+\frac{\alpha \widehat{w}(k)}{\tau_{R}\left|\dot{v}\left(T_{i}\right)\right|} \delta v\left(T_{i}^{-}\right), \quad i=1,2 .
$$



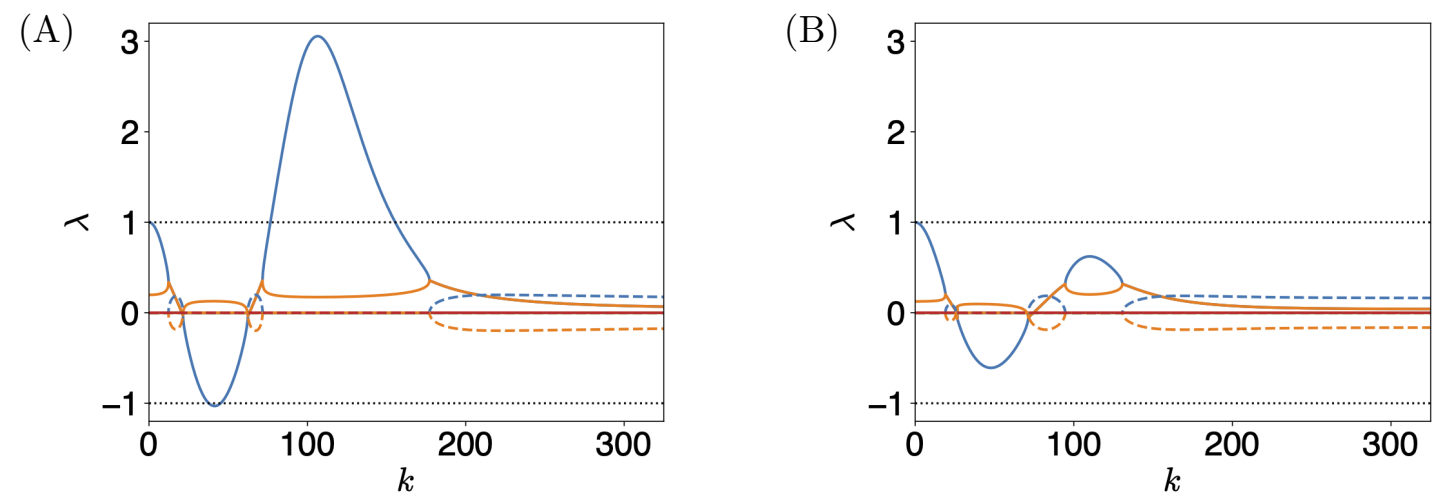

Figure 5: Real (solid lines) and imaginary (dashed lines) parts of the four eigenvalues of $\Psi(k)$ as a function of $k$ in blue, orange, green and red. The dotted black line delineates the stability boundary at $|\lambda|=1$. Parameter values as in Table 1 and (b) $g_{\mathrm{T}}=12.6 \mathrm{mVmS} / \mathrm{cm}^{2}, \gamma=0.65$.

As (3.7) relates perturbations before the switching event at times $T_{i}^{-}$to the value of $\delta r$ after the switching event at time $T_{i}^{+}$, we can read off the components for the saltation matrices $K_{1}$ and $K_{2}$ that pertain to $\delta r$. Because of the ansatz for $\delta z(x, t)$ shown after (3.3), the saltation matrices do not depend on space. They correspond to the prefactors of $\delta r\left(T_{i}^{-}\right)$and $\delta v\left(T_{i}^{-}\right)$, respectively. Note that at times $T_{3}$ and $T_{4}$ the dynamics of $r(x, t)$ changes continuously. Hence, the third row of $K_{3}$ and $K_{4}$ is identical to that of $I_{4}$. For convenience, we list all four saltation matrices in Appendix A.

Between switching events, the dynamics of $\delta Z(t)$ is governed by the linear system $\mathrm{d} \delta Z / \mathrm{d} t=$ $J_{i} z$, where $J_{i} \in \mathbb{R}^{4 \times 4}, i=1, \ldots, 4$, is piecewise constant as shown in Appendix A. Therefore, given an initial perturbation $\delta Z(0)$, the perturbation after one period $T$ equals $\delta Z(T)=$ $\Psi(k) \delta Z(0)$ with

$$
\Psi(k)=K_{4} \exp \left(J_{4} \Delta_{4}\right) K_{3} \exp \left(J_{3} \Delta_{3}\right) K_{2}(k) \exp \left(J_{2} \Delta_{2}\right) K_{1}(k) \exp \left(J_{1} \Delta_{1}\right) .
$$

We explicitly note the dependence on $k$ to highlight that $K_{1}$ and $K_{2}$ are functions of the wavenumber $k$. Hence, the synchronous state is linearly stable if the eigenvalues of $\Psi(k)$ (denoted $\left.\lambda_{i}, i=1, \ldots, 4\right)$ are contained within the unit disk for all $k$.

In Figure 5, we plot the eigenvalues of $\Psi(k)$ as a function of $k$ for two different sets of parameter values. In Figure 5A one of the eigenvalues is larger than 1 for a subset of wavenumbers, indicating that the synchronous solution is linearly unstable in this parameter regime. This is confirmed by numerical simulations shown in Figure 6A, where we depict the spatio-temporal evolution of $\delta v(x, t)$. Starting from a perturbed synchronous state with an unstable mode with wavenumber $k=106$, we find that perturbations grow. For the second set of parameter values, we observe in Figure $5 \mathrm{~B}$ that the eigenvalues remain inside the unit disc for all $k$. This is confirmed in Figure $6 \mathrm{~B}$ via direct simulation, where an initial perturbation around the synchronous state decays.

The propagator $\Psi(k)$ depends on $k$ only through the Fourier transform $\widehat{w}(k)$ of the connectivity kernel. Consequentially, changing the kernel may change the linear stability of the 

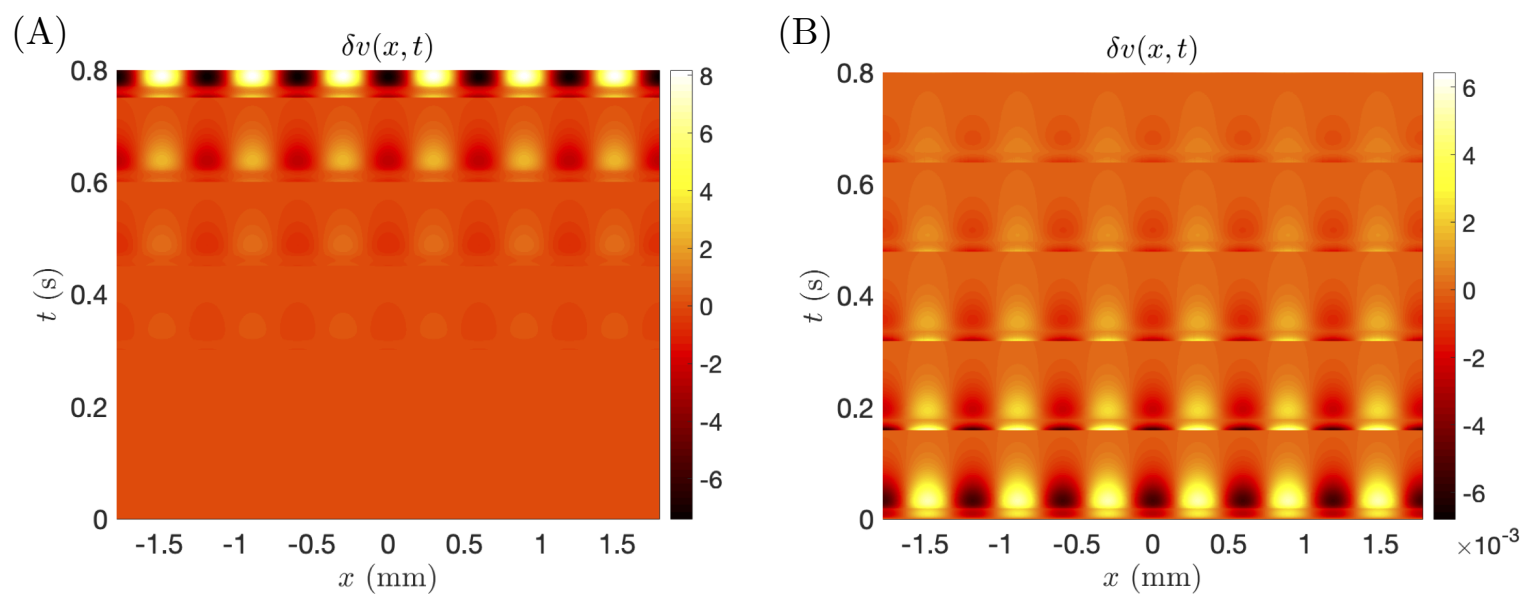

Figure 6: Space-time plot of the perturbation $\delta v(x, t)$ in $\mathrm{mV}$ when synchrony is linearly unstable (A) and stable (B). Parameters values as in the corresponding panels in Figure 5.
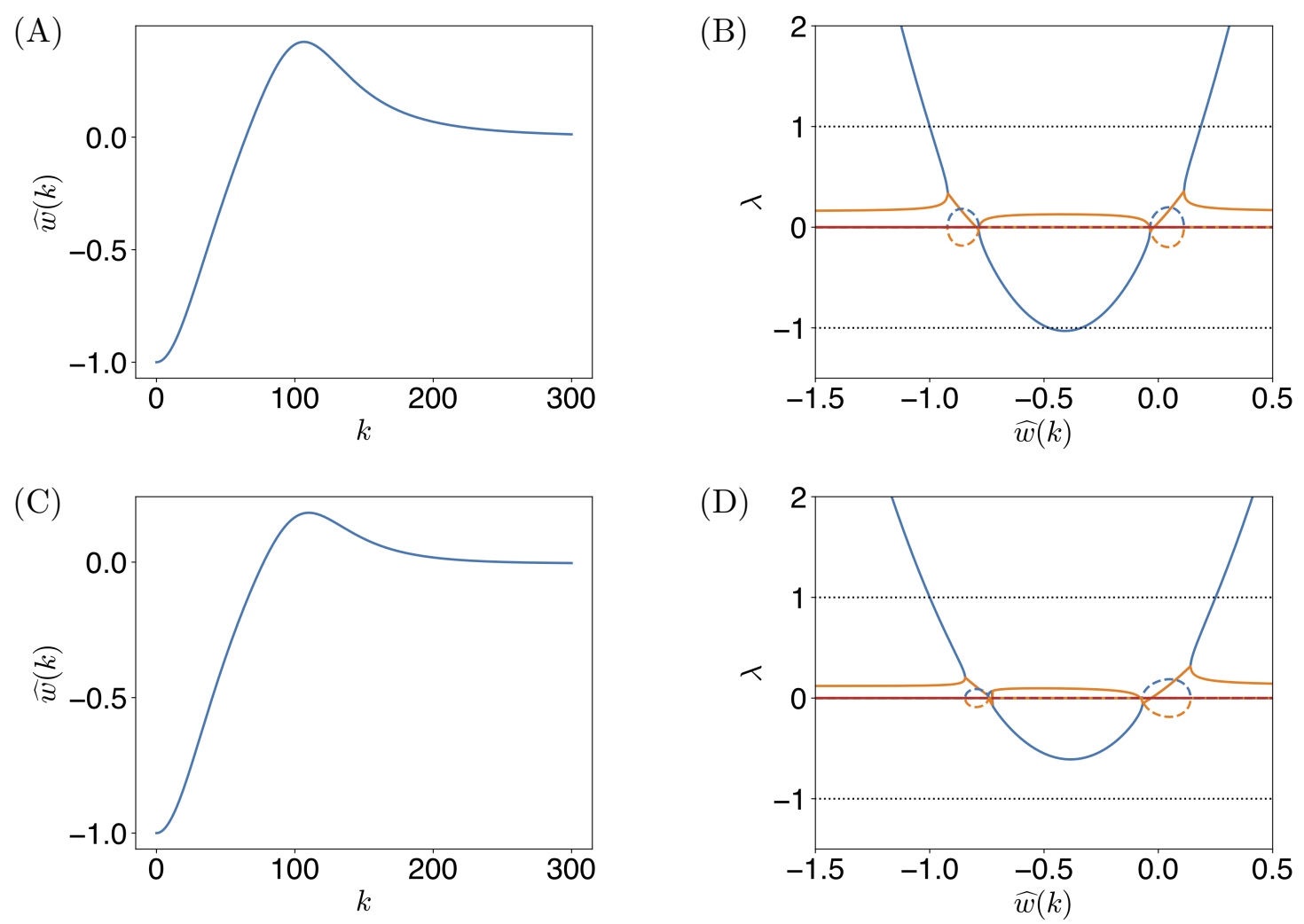

Figure 7: Fourier transform (left) and eigenvalues of $\Psi(k)$ as a function of $\widehat{w}(k)$ (right) for the set of standard parameter values (top) and when $g_{\mathrm{T}}=12.6 \mathrm{mVmS} / \mathrm{cm}^{2}$, and $\gamma=0.65$ (bottom). 


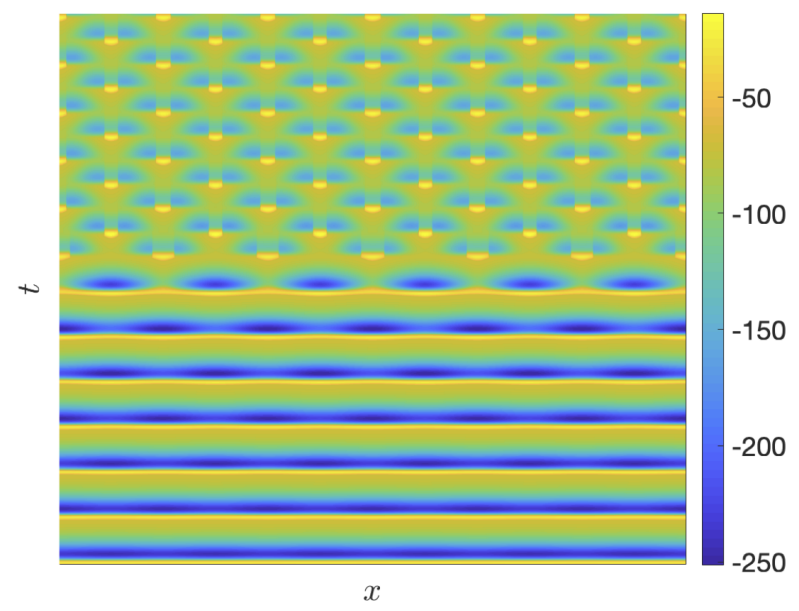

Figure 8: Space-time plot of the $v$ component in $\mathrm{mV}$ where an eigenvalue of $\Psi(k)$ leaves the unit disc along the real axis at -1 . Parameter values as in Table 1 and $\gamma=0.55, L=0.40 \mathrm{~cm}$.

synchronous state. This is illustrated in Figure 7, where we plot the eigenvalues of $\Psi(k)$ as a function of $\widehat{w}(k)$ for the two sets of parameter values in Figure 5. If the values of $\widehat{w}(k)$ are such that the corresponding eigenvalues are bounded between -1 and 1 , then synchrony is stable. Therefore, given the Fourier transform of a specific kernel, we only need to consult the appropriate plot of $\lambda_{i}, i=1, \ldots, 4$, as a function of $\widehat{w}(k)$ to infer linear stability of the synchronous state. As an example, consider the kernel in (2.6) with Fourier transform

$$
\widehat{w}(k)=-\left[a(k ; \sigma, 0)-\frac{\gamma}{2}(a(k ; \sigma, \rho)+a(k ; \sigma,-\rho))\right], \quad a(k ; \sigma, \rho)=\frac{2 \sigma}{1+(\rho-k \sigma)^{2}} .
$$

For the parameter values as in Figure $5 \mathrm{~A}$, we obtain $-1 \leq \widehat{w}(k) \leq 0.4235$ (see Figure 7A). We deduce from Figure 7B that synchrony is linearly unstable, which agrees with our previous result. On the contrary, the parameter values used in Figure 5B lead to the Fourier transform as shown in Figure $7 \mathrm{C}$ with $-1<\widehat{w}(k)<0.182$. Then Figure $7 \mathrm{D}$ shows synchrony is stable.

In the case where an eigenvalue of $\Psi(k)$ leaves the unit disc along the real axis at -1 then the synchronous solution breaks into standing oscillations in which a point in space fires on every other cycle, and the pattern on one cycle is shifted half a spatial cycle on the next temporal cycle. An example for this is shown in Figure 8. Where an eigenvalue of $\Psi(k)$ leaves the unit disc away from \pm 1 then quasi-periodic, complex periodic, and possibly chaotic solutions are expected to appear. We have not seen this type of instability of the synchronous state using realistic parameter values.

When probing the linear stability of the synchronous state in two spatial dimensions, the only change to the analysis above is that $\widehat{w}(k)$ is replaced by the two dimensional Fourier transform $\widehat{w}(\mathbf{k})=\int_{\mathbb{R}^{2}} w(\mathbf{r}) \mathrm{e}^{i \mathbf{k} \cdot \mathbf{r}} \mathrm{d} \mathbf{r}$ with $\mathbf{k} \in \mathbb{R}^{2}$. For the radially symmetric kernel given by (2.6) we have that

$$
\widehat{w}(k)=-[a(k ; \sigma, 0)-\gamma \operatorname{Re} a(k ; \sigma, \rho)], \quad a(k ; \sigma, \rho)=2 \pi \frac{1-i \rho}{\sigma\left[k^{2}+(1-i \rho)^{2} / \sigma^{2}\right]^{3 / 2}},
$$


where $k=|\mathbf{k}|$. Additional movie 3 in the Supplementary Material shows a 2D simulation when an eigenvalue crosses the unit disk along the real axis at -1 . Analogously to Figure 8 , we observe a period-doubling pattern, but this time the pattern is only transient before undergoing a secondary instability.

4. Periodic travelling waves. Travelling waves have been seen in a variety of different models of cortical and sub-cortical tissue as well as in vitro and in vivo [20]. Notable studies of travelling waves in thalamic models can be found in [25], which considers a biophysical cellbased model, and [31], which uses geometric singular perturbation methods to determine when waves exist. In the following we construct spatially periodic waves in one spatial dimension and determine the corresponding dispersion relation. Let $\xi=x-c t$ denote the co-moving variable. For ease of presentation, we use the same symbols for the periodic travelling wave as for the synchronous solution and differentiate between them through their arguments, i.e. $z(t)$ vs $z(\xi)$. Transforming the synaptic variable, $u(x, t)$, into the co-moving frame, using (2.4) and (2.5) as well as dropping the time dependence, we find

$$
u(\xi)=\frac{1}{\tau_{R}} \int_{-\infty}^{\infty} \mathrm{d} y w(|y|) \int_{0}^{\infty} \mathrm{d} s \eta(s) H\left(v(\xi-y+c s)-v_{\text {th }}\right) .
$$

For $\xi \in[0, \phi]$, where $\phi$ denotes the spatial period of the travelling wave in the co-moving frame, $v(\xi)$ exhibits an orbit that is topologically identical to the one shown in Figure 4. Hence, we can map the switching times $T_{i}$ to switching events at $\xi_{i}, i=1, \ldots, 4$, in the co-moving frame, where $\xi_{4}=\phi$ is the full spatial period, which entails that $v(0)=v_{\mathrm{h}}$. With this choice, the Heaviside function in (4.1) only contributes if $\xi_{1}+m \phi \leq \xi-y+c s \leq \xi_{2}+m \phi$ with $m \in \mathbb{Z}$. This reduces (4.1) to

$$
u(\xi)=\frac{1}{\tau_{R}} \int_{0}^{\infty} \mathrm{d} s \eta(s) \sum_{m \in \mathbb{Z}} \int_{\xi-\xi_{2}+c s-m \phi}^{\xi-\xi_{1}+c s-m \phi} \mathrm{d} y w(|y|),
$$

which can be rewritten as

$$
u(\xi)=\frac{1}{\tau_{R}} \sum_{m \in \mathbb{Z}} \int_{0}^{\infty} \mathrm{d} s \eta(s) W(-m \phi+c s+\xi),
$$

where

$$
W(x)=\int_{\xi_{1}}^{\xi_{2}} \mathrm{~d} y w(|x-y|) .
$$

It is now natural to express $u(\xi)$ as a Fourier series, which we obtain as

$$
u(\xi)=\sum_{p \in \mathbb{Z}} u_{p} \mathrm{e}^{2 \pi i p \xi / \phi}, \quad u_{p}=\frac{1}{\tau_{R} \phi} \widehat{\eta}\left(\frac{-2 \pi c p}{\phi}\right) \widehat{W}\left(\frac{2 \pi p}{\phi}\right) .
$$

Here, $\widehat{\eta}(k)$ and $\widehat{W}(k)$ denote the Fourier transforms of $\eta(t)$ and $W(x)$, respectively, which are given by

$$
\widehat{\eta}(k)=\left(\frac{\alpha}{\alpha+i k}\right)^{2}
$$


346 347

and

$$
\widehat{W}(k)=-\left[a(k, \sigma, 0)-\frac{\gamma}{2}(a(k, \sigma, \rho)+a(k, \sigma,-\rho))\right], \quad a(k ; \sigma, \rho)=\frac{2 \sigma i\left(e^{-i k \xi_{2}}-e^{-i k \xi_{1}}\right)}{k\left(1+(\rho-k \sigma)^{2}\right)}
$$

To determine the voltage profile $v(\xi)$, we transform (2.2) into the travelling wave frame, which results in

$$
-c \frac{\mathrm{d}}{\mathrm{d} \xi} v=g_{\mathrm{L}}\left(v_{\mathrm{L}}-v\right)+g_{\mathrm{T}} h H\left(v-v_{\mathrm{h}}\right)+g_{\mathrm{syn}} u .
$$

When we introduce the Green's function $G(\xi)=\mathrm{e}^{g_{\mathrm{L}} \xi / c}$ and recall that $v(0)=v_{\mathrm{h}}$, we can write a solution to (4.8) succinctly as

$$
v(\xi)=G(\xi) v_{\mathrm{h}}-\int_{0}^{\xi} G\left(\xi-\xi^{\prime}\right)\left(\frac{g_{\mathrm{L}} v_{L}}{c}+\frac{g_{\mathrm{T}}}{c} h\left(\xi^{\prime}\right) H\left(\xi_{3}-\xi\right)+\frac{g_{\mathrm{syn}}}{c} u\left(\xi^{\prime}\right)\right) \mathrm{d} \xi^{\prime},
$$

where the Heaviside function reflects the fact the $I_{T}$ is only present for $v>v_{\mathrm{h}}$, which is equivalent to $0 \leq \xi \leq \xi_{3}$. To compute $v(\xi)$, we require an expression for $h(\xi)$. This is readily achieved by transforming (2.3) into the travelling wave frame, which results in

$$
-c \frac{\mathrm{d}}{\mathrm{d} \xi} h=\frac{h_{\infty}(v)-h}{\tau_{h}(v)} .
$$

Note that (4.10) is an uncoupled piecewise linear equation, rendering its solution straightforward. Analogously to section 3 where we had to determine the switching times $T_{i}$ and the initial state $z_{0}$ to construct the synchronous solution, the periodic wave is parameterised by the switching coordinates $\xi_{i}, i=1,2,3$, the period $\phi$, the initial value $h(0)$ and the wave speed $c$. Since there are 6 unknowns, but only five conditions $\left(v\left(\xi_{1}\right)=v_{\mathrm{th}}, v\left(\xi_{2}\right)=v_{\mathrm{th}}, v\left(\xi_{3}\right)=v_{\mathrm{h}}\right.$, $\left.v(\phi)=v_{\mathrm{h}}, h(\phi)=h(0)\right)$, the wave speed $c$ becomes a function of the period $\phi$. Figure 9 shows the resultant dispersion relation for three different values of $\alpha$. As we decrease $\alpha$ going from the red to the black to the blue line, the emergent wave speed decreases. This is consistent with the interpretation of $\alpha$ as the inverse of the synaptic time scale. Decreasing $\alpha$ increases the time until peak synaptic response, which in turn results in synaptic activity spreading more slowly. The dots are results from numerical simulation and agree very well with our theoretical predictions. We already indicate linear stability of the periodic travelling waves in Figure 9. Linearly stable travelling waves are denoted by solid lines, while dashed lines refer to linearly unstable travelling waves. When initiating a numerical simulation with an unstable pattern, the emergent travelling wave is selected from the possible periods in the stable region of Figure 9. As an example, we initiated a travelling wave with a spatial period of $\phi=1.32 \mathrm{~mm}$, which is linearly unstable. The emergent wave has a spatial period of $\phi=0.66 \mathrm{~mm}$ and is shown by an asterisks. The corresponding space-time plot of the periodic travelling wave is depicted in Figure 10.

5. Stability of travelling waves. Having constructed periodic travelling waves in section 4 , we already indicated in the dispersion relation in Figure 9 the linear stability of these waves. Those results are based on the following linear stability analysis. Let $\delta z(\xi, t)$ denote a 


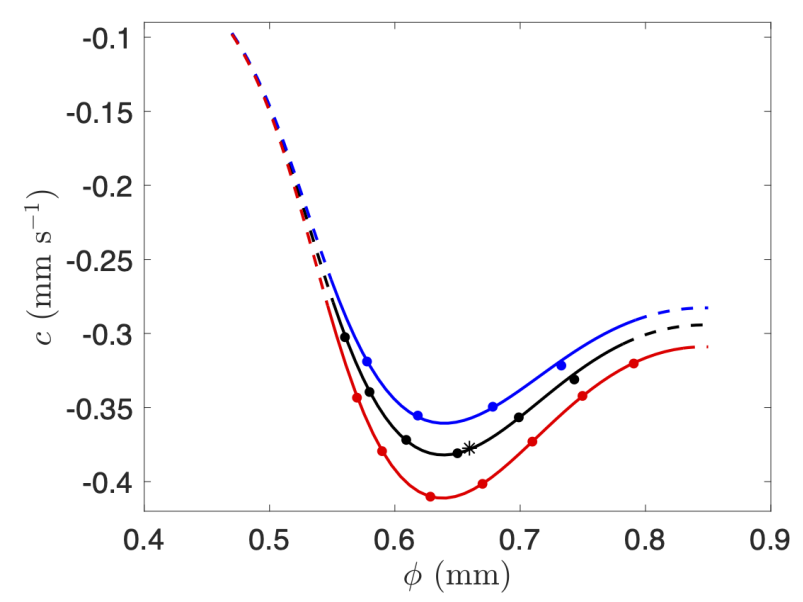

Figure 9: Dispersion curves $c=c(\phi)$ for periodic travelling waves for three different values of $\alpha$ : $0.07 \mathrm{~ms}^{-1}$ (blue), $0.1 \mathrm{~ms}^{-1}$ (black) and $0.2 \mathrm{~ms}^{-1}$ (red). Solid lines indicate linearly stable travelling waves, while dashed lines correspond to linearly unstable travelling waves. The Evans function plots in Figures 11 to 13 demonstrate how we have delineated the stability boundaries for the three dispersion curves plotted. See the main body of text in section 5 for further details. Dots indicate simulation results. The asterisk represents the emergent wave speed and period of an initially unstable wave with period $\phi=1.32 \mathrm{~mm}$, with the spatiotemporal plot showing the wavetrain instability and eventual emergent pattern shown in Figure 10. Parameter values as in Table 1.

perturbation around the periodic travelling wave $z(\xi)$. We again use the same notation for the perturbation of the periodic travelling wave as we did for those of the synchronous solution along the lines introduced in section 4. Based on (4.1), we obtain for the perturbation in synaptic activity

$$
\delta u(\xi, t)=\frac{1}{\tau_{R}} \int_{-\infty}^{\infty} \mathrm{d} y w(|y|) \int_{0}^{\infty} \mathrm{d} s \eta(s) \sum_{m \in \mathbb{Z}} \sum_{i=1}^{2} \frac{\delta\left(\xi-y+c s-m \phi-\xi_{i}\right)}{\left|v^{\prime}\left(\xi_{i}\right)\right|} \delta v(\xi-y+c s, t-s),
$$

where we used the fact that the voltage $v(\xi)$ crosses $v_{\text {th }}$ in the travelling wave frame at $\xi_{1}+m \phi$ and $\xi_{2}+m \phi$, respectively, as well as that $v^{\prime}(m \phi+\xi)=v^{\prime}(\xi)$. Here, $m \in \mathbb{Z}$, and the prime indicates differentiation with respect to $\xi$. We now make the ansatz that $\delta z(\xi, t)=\delta Z(\xi) \mathrm{e}^{\lambda t}$ and assume that $\delta Z(\xi)$ is $\phi$-periodic, i.e. $\delta Z(\xi+\phi)=\delta Z(\xi)$. This results in

$$
\delta u(\xi ; \lambda)=\frac{1}{\tau_{R}} \sum_{m \in \mathbb{Z}} \int_{0}^{\infty} \mathrm{d} s \eta(s) \mathrm{e}^{-\lambda s} \sum_{i=1}^{2} w\left(\left|\xi-\xi_{i}-m \phi+c s\right|\right) \frac{\delta v\left(\xi_{i}\right)}{\left|v^{\prime}\left(\xi_{i}\right)\right|}
$$

As we did for the synaptic activity in section 4 , we express $\delta u(\xi)$ as a Fourier series such that

$$
\delta u(\xi)=\sum_{p \in \mathbb{Z}} u_{p} \mathrm{e}^{2 \pi i p \xi / \phi}, \quad u_{p}=\frac{1}{\tau_{R} \phi} \widehat{w}\left(\frac{2 \pi p}{\phi}\right) \sum_{q=1}^{2} I_{q},
$$




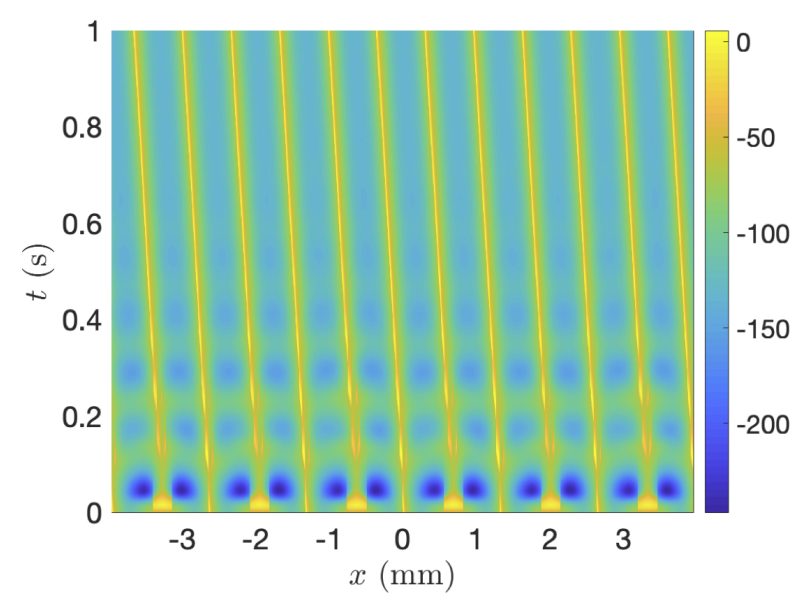

Figure 10: Space-time plot of the $v$ component in $\mathrm{mV}$ of the travelling wave corresponding to the asterisk in Figure 9.

where

$$
I_{q}=\frac{\delta v\left(\xi_{q}\right)}{\left|v^{\prime}\left(\xi_{q}\right)\right|} \widetilde{\eta}\left(\lambda-\frac{2 \pi i p c}{\phi}\right) \mathrm{e}^{-2 \pi i p \xi_{q} / \phi}
$$

and

$$
\widetilde{\eta}(k)=\int_{0}^{\infty} \eta(s) \mathrm{e}^{-k s} \mathrm{~d} s,
$$

represents the Laplace transform of $\eta(s)$. To obtain solutions for $\delta v(\xi)$ and $\delta h(\xi)$ we transform (2.2) and (2.3) into the travelling wave frame. Recalling the exponential time dependence of $\delta z(\xi, t)$, we find that

$$
c \frac{\mathrm{d}}{\mathrm{d} \xi} \delta v(\xi)= \begin{cases}\left(g_{\mathrm{L}}+\lambda\right) \delta v(\xi)+g_{\mathrm{T}} \delta h(\xi)+g_{\mathrm{syn}} \delta u(\xi), & 0^{+} \leq \xi \leq \xi_{3}^{-} \\ \left(g_{\mathrm{L}}+\lambda\right) \delta v(\xi)+g_{\mathrm{syn}} \delta u(\xi), & \xi_{3}^{+} \leq \xi \leq \phi^{-}\end{cases}
$$

and

$$
c \frac{\mathrm{d}}{\mathrm{d} \xi} \delta h(\xi)= \begin{cases}\left(\frac{1}{\tau_{\mathrm{h}}^{-}}+\lambda\right) \delta h(\xi), & 0^{+} \leq \xi \leq \xi_{3}^{-}, \\ \left(\frac{1}{\tau_{\mathrm{h}}^{+}}+\lambda\right) \delta h(\xi), & \xi_{3}^{+} \leq \xi \leq \phi^{-} .\end{cases}
$$

Equations (5.6) and (5.7) are readily solved via the Green's functions $G_{v}(\xi)=\mathrm{e}^{\left(g_{\mathrm{L}}+\lambda\right) \xi / c}$ and $G_{h}^{ \pm}(\xi)=\mathrm{e}^{\left(1 / \tau_{h}^{ \pm}+\lambda\right) \xi / c}$. For $0^{+} \leq \xi \leq \xi_{3}^{-}$, we obtain

$$
\delta v(\xi)=G_{v}(\xi) \delta v\left(0^{+}\right)+\int_{0}^{\xi} G_{v}\left(\xi-\xi^{\prime}\right)\left(\frac{g_{\mathrm{T}}}{c} \delta h\left(\xi^{\prime}\right)+\frac{g_{\mathrm{syn}}}{c} \delta u\left(\xi^{\prime}\right)\right) \mathrm{d} \xi^{\prime},
$$

$$
\delta h(\xi)=G_{h}^{-}(\xi) \delta h\left(0^{+}\right),
$$


407

408

498

while for $\xi_{3}^{+} \leq \xi \leq \phi^{-}$, we arrive at

$$
\begin{aligned}
& \delta v(\xi)=G_{v}\left(\xi-\xi_{3}\right) \delta v\left(\xi_{3}^{+}\right)+\frac{g_{\mathrm{syn}}}{c} \int_{\xi_{3}}^{\xi} G_{v}\left(\xi-\xi^{\prime}\right) \delta u\left(\xi^{\prime}\right) \mathrm{d} \xi^{\prime}, \\
& \delta h(\xi)=G_{h}^{+}\left(\xi-\xi_{3}\right) \delta h\left(\xi_{3}^{+}\right) .
\end{aligned}
$$

Note that the solutions in (5.8) and (5.9) depend on the variables evaluated at $0^{+}$and $\xi_{3}^{+}$, respectively. This results from the non-smooth dynamics of $v$ and $h$ when the voltage crosses $v_{\mathrm{h}}$. Analogously to section 3 , we require saltation matrices $Q_{i}$ to propagate perturbations through the switching events: $\delta Z_{r}\left(\xi_{i}^{+}\right)=Q_{i} \delta Z_{r}\left(\xi_{i}^{-}\right)$, where we have introduced the reduced state vectors $Z_{r}(\xi)=(v(\xi), h(\xi))$ and $\delta Z_{r}(\xi)=(\delta v(\xi), \delta h(\xi))$. Following [3], the saltation matrices $Q_{i}$ have the same form as in (3.2), with $I_{4}$ replaced by $I_{2}$ and $F_{i}^{ \pm}$constructed from the equations that govern the periodic solution derived in section 4 , i.e.

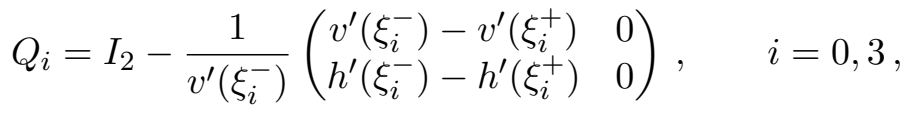

where we set $\xi_{0}=0$. Since we consider $\phi$-periodic perturbations, we have $\delta Z_{r}\left(0^{+}\right)=$ $Q_{0} \delta Z_{r}\left(\phi^{-}\right)$. Using (5.8) and (5.9), it is therefore possible to express $\delta Z_{r}\left(0^{+}\right)$as a linear combination of $\delta v\left(\xi_{1}\right), \delta v\left(\xi_{2}\right), \delta v\left(\xi_{3}^{-}\right)$and $\delta v\left(\phi^{-}\right)$. As an illustration, we show the calculation for $\delta h\left(0^{+}\right)$in Appendix A. When we evaluate $\delta v(\xi)$ at $\xi_{1}, \xi_{2}, \xi_{3}^{-}$and $\xi_{4}^{-}$, we obtain the linear system $\left(\Gamma(\lambda)-I_{4}\right) \mathbf{x}=0$, where

$$
\Gamma(\lambda)=\left(\begin{array}{llll}
f_{1}\left(\xi_{1} ; \lambda\right) & f_{2}\left(\xi_{1} ; \lambda\right) & f_{3}\left(\xi_{1} ; \lambda\right) & f_{4}\left(\xi_{1} ; \lambda\right) \\
f_{1}\left(\xi_{2} ; \lambda\right) & f_{2}\left(\xi_{2} ; \lambda\right) & f_{3}\left(\xi_{2} ; \lambda\right) & f_{4}\left(\xi_{2} ; \lambda\right) \\
f_{1}\left(\xi_{3} ; \lambda\right) & f_{2}\left(\xi_{3} ; \lambda\right) & f_{3}\left(\xi_{3} ; \lambda\right) & f_{4}\left(\xi_{3} ; \lambda\right) \\
g_{1}\left(\xi_{4} ; \lambda\right) & g_{2}\left(\xi_{4} ; \lambda\right) & g_{3}\left(\xi_{4} ; \lambda\right) & g_{4}\left(\xi_{4} ; \lambda\right)
\end{array}\right),
$$

and $\mathbf{x}=\left(\delta v\left(\xi_{1}\right), \delta v\left(\xi_{2}\right), \delta v\left(\xi_{3}^{-}\right), \delta v\left(\xi_{4}^{-}\right)\right)$. The entries of $\Gamma$ are listed in Appendix B. A nontrivial solution for $\mathbf{x}$ requires that the Evans function

$$
\mathcal{E}(\lambda)=\operatorname{det}\left(\Gamma(\lambda)-I_{4}\right),
$$

vanishes. This only happens at certain values of $\lambda$. Since in general $\lambda \in \mathbb{C}$, we set $\lambda=a+i b$ and then compute the zero-contours of $\operatorname{Re} \mathcal{E}(\lambda)$ and $\operatorname{Im} \mathcal{E}(\lambda)$. Their intersections signify a zero of $\mathcal{E}$ and hence an admissible value for $\lambda$. When all eigenvalues have negative real part, the periodic travelling wave is stable, otherwise, it is unstable.

Figure 11 shows the case when all eigenvalues are contained in the left-hand side of the complex plane and hence the periodic travelling wave is linearly stable. Upon increasing $\phi$ a pair of eigenvalues touches the imaginary axis (Figure 12) indicating the onset of an instability. This point is shown in Figure 9 by a transition from a black solid to a black dashed line at $\phi=0.782 \mathrm{~mm}$. For even larger values of $\phi$, a pair of eigenvalues with positive real parts exists (Figure 13), indicating a linearly unstable periodic travelling wave. In Figures 11 - 13, there is always an eigenvalue at zero, which originates from the translational invariance of the underlying equations. The persistence of the translation invariance zero eigenvalue can be 

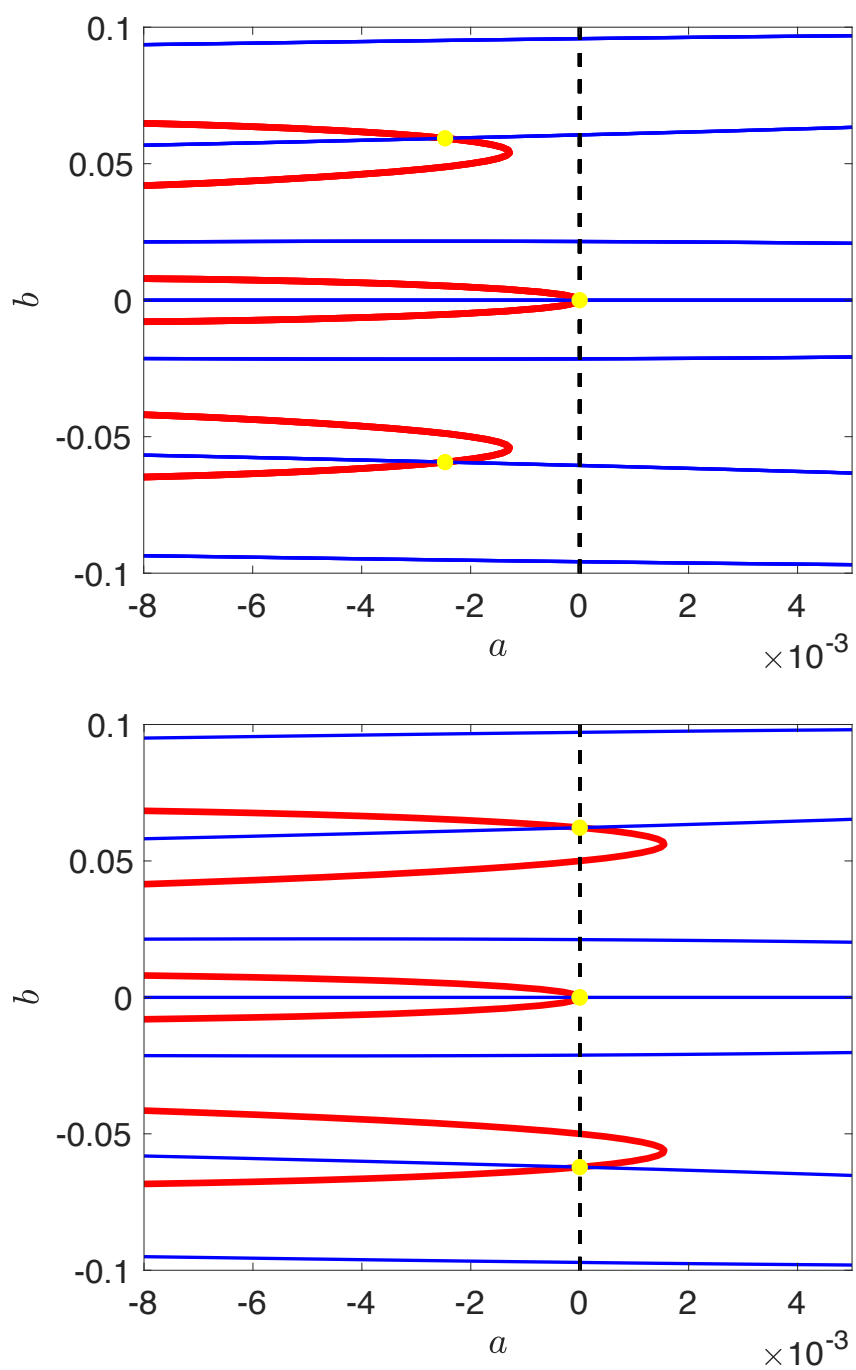

Figure 11: Zero-contours of $\operatorname{Re} \mathcal{E}(\lambda)$ (thick, red curve) and of $\operatorname{Im} \mathcal{E}(\lambda)$ (thin, blue curve) for $\phi=0.74 \mathrm{~mm}$. Intersections of the zero-contours, and hence zeroes of $\mathcal{E}(\lambda)$, are shown as yellow dots. The black dashed line separates the two complex half planes with negative and positive real part, respectively. Parameter values as in Table 1.

Figure 12: Zero-contours of $\operatorname{Re} \mathcal{E}(\lambda)$ (thick, red curve) and of $\operatorname{Im} \mathcal{E}(\lambda)$ (thin, blue curve) for $\phi=0.782 \mathrm{~mm}$. Intersections of the zero-contours, and hence zeroes of $\mathcal{E}(\lambda)$, are shown as yellow dots. The black dashed line separates the two complex half planes with negative and positive real part, respectively. Parameter values as in Table 1.

established by showing that $z^{\prime}(\xi)$ is an eigenfunction to the stability problem when $\lambda=0$. For the local variables, $v(\xi)$ and $h(\xi)$, this is readily shown by differentiating the travelling wave equations (4.8) and (4.10) with respect to $\xi$ and comparing with (5.6) and (5.7), respectively. In the case of the synaptic variable, first differentiate (4.3) to obtain

$$
\frac{\mathrm{d} u}{\mathrm{~d} \xi}=\frac{1}{\tau_{R}} \sum_{m \in \mathbb{Z}} \int_{0}^{\infty} \mathrm{d} s \eta(s)\left(w\left(\left|-m \phi+c s+\xi-\xi_{1}\right|\right)-w\left(\left|-m \phi+c s+\xi-\xi_{2}\right|\right)\right),
$$

Then setting $\lambda=0$ in (5.2), making the substitution $\delta Z(\xi)=z^{\prime}(\xi)$, and noting that $v^{\prime}\left(\xi_{1}\right)>0$ and $v^{\prime}\left(\xi_{2}\right)<0$, one obtains (5.13). Therefore, $\delta Z(\xi)=z^{\prime}(\xi)$ is a solution to the stability problem when $\lambda=0$, showing the translation invariance of the system, and that tangential perturbations to the orbit are neutrally stable.

6. Discussion. Neural field models are ubiquitous throughout the computational and mathematical neuroscience community for their use in understanding the waves and pat- 
Figure 13: Zero-contours of $\operatorname{Re} \mathcal{E}(\lambda)$ (thick, red curve) and of $\operatorname{Im} \mathcal{E}(\lambda)$ (thin, blue curve) for $\phi=0.824 \mathrm{~mm}$. Intersections of the zero-contours, and hence zeroes of $\mathcal{E}(\lambda)$, are shown as yellow dots. The black dashed line separates the two complex half planes with negative and positive real part, respectively. Parameter values as in Table 1.

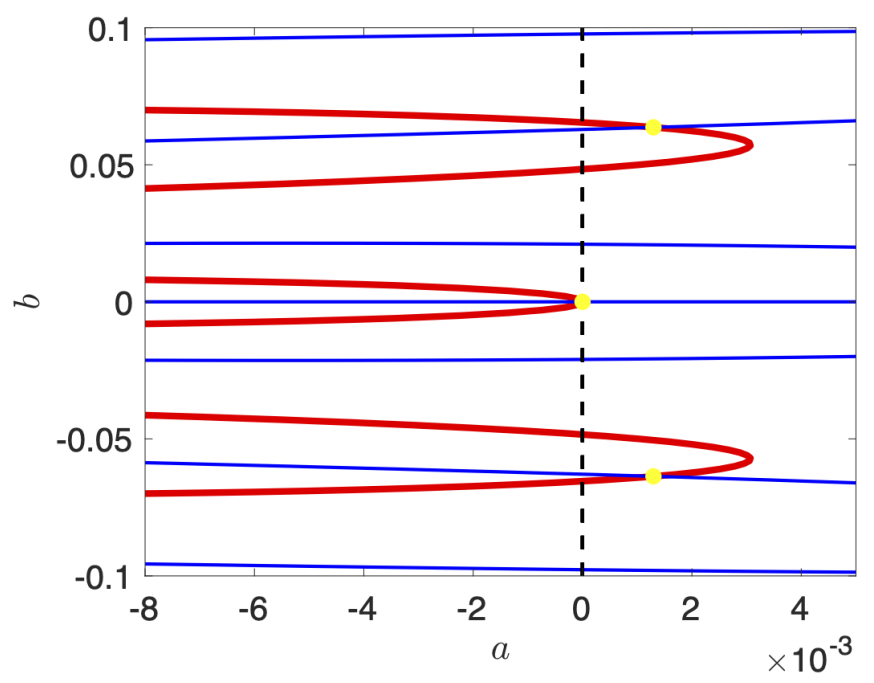

terns that are readily observed in cortex. For example, voltage-sensitive dyes can be used to observe waves of activity in the primary visual cortex of the awake monkey [21], and electro-encephalograms routinely exhibit alpha oscillations propagating over the human scalp [14]. Nonetheless, their domain of applicability does not immediately extend to modelling sub-cortical structures without due consideration of how this tissue may differ from that of the cortex. A case in point is the thalamus where nonlinear ionic currents expressed at the cellular level play a large role in shaping spatio-temporal patterns seen at the tissue level [9]. This has been explored from a theoretical perspective by Rinzel et al. [25] using a computational model, and by Yew et al. [31] from a more mathematical perspective using singular perturbation methods. However, analytical progress on waves in such sub-cortical structures has not kept pace with the growth of results for standard neural fields, as reviewed in [7]. This is in part because of certain cortical model simplifications, and in particular the "Heaviside world" perspective promoted by Amari [2]. Here, we have shown that the switch-like dynamics governing many neuronal ionic currents can be accommodated within a standard Amari style neural field model by augmenting the Heaviside firing rate to be a function of a (non-spiking) voltage variable that tracks the gating dynamics for sub-cellular currents. If the latter is caricatured by activation or inactivation functions that are piece-wise constant (step functions) then the Amari programme for finding closed form solutions and determining their stability goes over albeit with the recognition that one is now dealing with a non-smooth system. To illustrate how this programme can be applied in practice, we have considered the inclusion of a slow T-type calcium current into a continuum model of an inhibitory network, in the spirit of that employed by Huertas et al. [15] as a minimal model of the thalamus (dorsal lateral geniculate nucleus). The model supports robust whole tissue synchronous oscillations via the mechanism of post-inhibitory rebound for a wide range of parameters. By merging the traditional Amari approach with tools from nonsmooth dynamical systems, we have shown how to construct new network level saltation operators to determine the linear stability of the synchronous solution. We have used this to showcase the possibility of emergent solutions which have a spatially periodic component (with a wavelength determined by the Fourier transform 
of the anatomical connectivity pattern) superimposed on the bulk oscillation. Moreover, direct simulations also show that the model can support more exotic solutions, in the form of both regular and irregular travelling waves. By moving to a co-moving frame we have shown how to construct the former (in one spatial dimension), and made use of saltation operators to construct the Evans function for periodic travelling waves. Direct numerical simulations show excellent agreement with the stable branch of the theoretically determined dispersion curve.

The mathematical analysis presented in this paper was motivated by the novel patterns seen in direct simulations. Although we have taken a step toward understanding the potentially very rich dynamics that can arise in models of neural tissue with rebound currents, there is clearly much more to do in analysing secondary bifurcations and the routes to exotic pattern generation. This could include a more thorough analysis of planar waves (generalising what we have achieved here for waves in one spatial dimension), as well as exploring so-called lurching waves that are also a hallmark of systems with rebound currents [25]. It would also be natural to consider more refined models of the thalamus that include the two main cell types of reticular (RE) and thalamo-cortical (TC) relay cells and their reciprocal connections. Both express an $I_{\mathrm{T}}$ current though in a way that allows TC cells to fire upon release from inhibition and RE cells to burst in response to excitation. The model presented here is easily generalised to this case following the modelling approach described in [5]. Moreover, it is also possible to accommodate window currents that arise when multiple gating variables conspire to generate persistent background currents. The use of piecewise linear, rather than piecewise constant, activation and inactivation curves that overlap can account for this, without detriment to mathematical tractability [19]. All of these are topics of ongoing study and will be reported upon elsewhere. 
511 (A.1b)

512 (A.1c)

\section{Appendix A. Saltation matrices and Jacobians.}

The equations governing the synchronous solution (3.1) contain discontinuities in the vector field. To determine linear stability, we require saltation matrices that map perturbations across discontinuities. A derivation of saltation matrices for temporal switching events can be found in [22]. For all local dynamics, we are permitted to use the first, second and fourth rows of (3.2). For the non-local dynamics in the third rows of $K_{1}$ and $K_{2}$, we derive the correct expression as discussed in section 3. Using this approach, the saltation matrices read as

$K_{2}=\left(\begin{array}{cccc}0 & 1 & 0 & 0 \\ -\frac{\alpha}{\tau_{\mathrm{R}}} \frac{\widehat{w}(k)}{\dot{v}\left(T_{2}^{-}\right)} & 0 & 1 & 0 \\ 0 & 0 & 0 & 1\end{array}\right)$,

$$
\begin{aligned}
K_{1} & =\left(\begin{array}{cccc}
1 & 0 & 0 & 0 \\
0 & 1 & 0 & 0 \\
\frac{\alpha}{\tau_{\mathrm{R}}} \frac{\widehat{w}(k)}{\dot{v}\left(T_{1}^{-}\right)} & 0 & 1 & 0 \\
0 & 0 & 0 & 1
\end{array}\right), \\
K_{2} & =\left(\begin{array}{cccc}
1 & 0 & 0 & 0 \\
0 & 1 & 0 & 0 \\
-\frac{\alpha}{\tau_{\mathrm{R}}} \frac{\widehat{w}(k)}{\dot{v}\left(T_{2}^{-}\right)} & 0 & 1 & 0 \\
0 & 0 & 0 & 1
\end{array}\right),
\end{aligned}
$$

$$
\begin{aligned}
K_{3}= & \left(\begin{array}{cccc}
1-\frac{g_{\mathrm{T}} h\left(T_{3}^{-}\right)}{C \dot{v}\left(T_{3}^{-}\right)} & 0 & 0 & 0 \\
0 & 1 & 0 & 0 \\
0 & 0 & 1 & 0 \\
\frac{1 / \tau^{+}-h\left(T_{3}^{+}\right) / \tau^{+}+h\left(T_{3}^{-}\right) / \tau^{-}}{\dot{v}\left(T_{3}^{-}\right)} & 0 & 0 & 1
\end{array}\right), \\
K_{4}= & \left.0 \begin{array}{cccc}
1+\frac{g_{\mathrm{T}} h\left(0^{+}\right)}{C \dot{v}\left(T_{4}^{-}\right)} & 0 & 0 \\
0 & 1 & 0 & 0 \\
0 & 0 & 1 & 0 \\
\frac{-h\left(0^{+}\right) / \tau^{-}-1 / \tau^{+}+h\left(T_{4}^{-}\right) / \tau^{+}}{\dot{v}\left(T_{4}^{-}\right)} & 0 & 0 & 1
\end{array}\right) .
\end{aligned}
$$

The dynamics away from switching events is piecewise linear. Therefore, the propagator takes the form $\exp (J t)$, where $J$ is the piecewise constant Jacobian matrix and is given by

$$
J= \begin{cases}J_{1}, & 0^{+} \leq t \leq T_{1}^{-} \\ J_{2}, & T_{1}^{+} \leq t \leq T_{2}^{-} \\ J_{3}, & T_{2}^{+} \leq t \leq T_{3}^{-} \\ J_{4}, & T_{3}^{+} \leq t \leq T^{-}\end{cases}
$$


where

$$
\begin{aligned}
J_{1}=J_{2}=J_{3} & =\left(\begin{array}{cccc}
-g_{\mathrm{L}} / C & g_{\mathrm{syn}} / C & 0 & g_{\mathrm{T}} / C \\
0 & -\alpha & \alpha & 0 \\
0 & 0 & -\alpha & 0 \\
0 & 0 & 0 & -1 / \tau^{-}
\end{array}\right), \\
J_{4} & =\left(\begin{array}{cccc}
-g_{\mathrm{L}} / C & g_{\mathrm{syn}} / C & 0 & 0 \\
0 & -\alpha & \alpha & 0 \\
0 & 0 & -\alpha & 0 \\
0 & 0 & 0 & -1 / \tau^{+}
\end{array}\right) .
\end{aligned}
$$

Whereas the synchronous solution is spatially homogeneous, periodic travelling waves are not, and therefore switching in the dynamics has a spatial dependence. See the Appendix of [3] for a derivation of the saltation matrix in the co-moving frame. In our case, the perturbed synapse variable is a smooth function of $\xi$, so saltation matrices in this case take the reduced form (5.10), where only $\delta v(\xi)$ and $\delta h(\xi)$ contain discontinuities. Let $V_{i}$ denote the $(2,1)$ component of $Q_{i}$ as defined in (5.10). Using (5.8b) and (5.9b), we find that

$$
\begin{aligned}
\delta h\left(0^{+}\right) & =V_{0} \delta v\left(\phi^{-}\right)+\delta h\left(\phi^{-}\right) \\
& =V_{0} \delta v\left(\phi^{-}\right)+G_{h}^{+}\left(\phi-\xi_{3}\right) \delta h\left(\xi_{3}^{+}\right) \\
& =V_{0} \delta v\left(\phi^{-}\right)+G_{h}^{+}\left(\phi-\xi_{3}\right)\left(V_{3} \delta v\left(\xi_{3}^{-}\right)+\delta h\left(\xi_{3}^{-}\right)\right) \\
& =V_{0} \delta v\left(\phi^{-}\right)+V_{3} G_{h}^{+}\left(\phi-\xi_{3}\right) \delta v\left(\xi_{3}^{-}\right)+G_{h}^{+}\left(\phi-\xi_{3}\right) G_{h}^{-}\left(\xi_{3}\right) \delta h\left(0^{+}\right),
\end{aligned}
$$

so that

$$
\delta h\left(0^{+}\right)=\frac{V_{0} \delta v\left(\phi^{-}\right)+V_{3} G_{h}^{+}\left(\phi-\xi_{3}\right) \delta v\left(\xi_{3}^{-}\right)}{1-G_{h}^{+}\left(\phi-\xi_{3}\right) G_{h}^{-}\left(\xi_{3}\right)} .
$$

\section{Appendix B. Functions for Evans function calculation.}

Here, we give the functions that are the entries of the matrix $\Gamma(\lambda),(5.11)$, which is required for computing the Evans function.

$$
\begin{aligned}
& f_{1}(\xi)=\frac{g_{\mathrm{syn}}}{c} \frac{1}{\left|v^{\prime}\left(\xi_{1}^{-}\right)\right|} \sum_{p \in \mathbb{Z}} \bar{I}_{p, \xi_{1}, \lambda}\left(\mathrm{e}^{\frac{2 \pi i p \xi}{\phi}}-\mathrm{e}^{\left(\frac{g_{\mathrm{L}}+\lambda}{c}\right) \xi}\right) \\
& f_{2}(\xi)=\frac{g_{\mathrm{syn}}}{c} \frac{1}{\left|v^{\prime}\left(\xi_{2}^{-}\right)\right|} \sum_{p \in \mathbb{Z}} \bar{I}_{p, \xi_{2}, \lambda}\left(\mathrm{e}^{\frac{2 \pi i p \xi}{\phi}}-\mathrm{e}^{\left(\frac{g_{\mathrm{L}}+\lambda}{c}\right) \xi}\right), \\
& f_{3}(\xi)=\frac{g_{\mathrm{T}}}{c} f_{h}(\xi)\left(-\frac{h^{\prime}\left(\xi_{3}^{-}\right)-h^{\prime}\left(\xi_{3}^{+}\right)}{v^{\prime}\left(\xi_{3}^{-}\right)} \mathrm{e}^{\left(\lambda / c+1 / c \tau^{+}\right)\left(\phi-\xi_{3}\right)}\right) \\
& f_{4}(\xi)=\left(1-\frac{v^{\prime}\left(\phi^{-}\right)-v^{\prime}\left(0^{+}\right)}{v^{\prime}\left(\phi^{-}\right)}\right) \mathrm{e}^{\left(\frac{g_{\mathrm{L}}+\lambda}{c}\right) \xi}-\frac{g_{\mathrm{T}}}{c} f_{h}(\xi)\left(\frac{h^{\prime}\left(\phi^{-}\right)-h^{\prime}\left(0^{+}\right)}{v^{\prime}\left(\phi^{-}\right)}\right),
\end{aligned}
$$


539

540

where

$$
\begin{aligned}
\bar{I}_{p, a, \lambda} & =\frac{I_{p, a, \lambda}}{\frac{2 \pi i p}{\phi}-\frac{g_{\mathrm{L}}+\lambda}{c}}, \\
I_{p, a, \lambda} & =\frac{1}{\tau_{R} \phi} \tilde{\eta}\left(\lambda-\frac{2 \pi i p c}{\phi}\right) \hat{w}\left(\frac{2 \pi p}{\phi}\right) \mathrm{e}^{-\frac{2 \pi i p a}{\phi}},
\end{aligned}
$$

and

$$
f_{h}(\xi)=\frac{\mathrm{e}^{\left(\frac{\lambda}{c}+\frac{1}{c \tau^{-}}\right) \xi}-\mathrm{e}^{\left(\frac{g_{\mathrm{L}}+\lambda}{c}\right) \xi}}{\left(\frac{\lambda}{c}+\frac{1}{c \tau^{-}}-\frac{g_{\mathrm{L}}+\lambda}{c}\right)\left(1-\mathrm{e}^{\left(\frac{\lambda}{c}+\frac{1}{c \tau^{-}}\right) \xi_{3}} \mathrm{e}^{\left(\frac{\lambda}{c}+\frac{1}{c \tau^{+}}\right)\left(\phi-\xi_{3}\right)}\right)},
$$

and

$$
g_{1}(\xi)=\frac{g_{\mathrm{syn}}}{c} \frac{1}{\left|v^{\prime}\left(\xi_{1}^{-}\right)\right|} \sum_{p \in \mathbb{Z}} \bar{I}_{p, \xi_{1}, \lambda}\left(\mathrm{e}^{\frac{2 \pi i p \xi}{\phi}}-\mathrm{e}^{\frac{2 \pi i p \xi_{3}}{\phi}} \mathrm{e}^{\left(\frac{g_{\mathrm{L}}+\lambda}{c}\right)\left(\xi-\xi_{3}\right)}\right),
$$

$$
g_{2}(\xi)=\frac{g_{\mathrm{syn}}}{c} \frac{1}{\left|v^{\prime}\left(\xi_{2}^{-}\right)\right|} \sum_{p \in \mathbb{Z}} \bar{I}_{p, \xi_{2}, \lambda}\left(\mathrm{e}^{\frac{2 \pi i p \xi}{\phi}}-\mathrm{e}^{\frac{2 \pi i p \xi_{3}}{\phi}} \mathrm{e}^{\left(\frac{g_{\mathrm{L}}+\lambda}{c}\right)\left(\xi-\xi_{3}\right)}\right) \text {, }
$$

$$
g_{3}(\xi)=\left(1-\frac{v^{\prime}\left(\xi_{3}^{-}\right)-v^{\prime}\left(\xi_{3}^{+}\right)}{v^{\prime}\left(\xi_{3}^{-}\right)}\right) \mathrm{e}^{\left(\frac{g_{\mathrm{L}}+\lambda}{c}\right)\left(\xi-\xi_{3}\right)},
$$

$$
g_{4}(\xi)=0 \text {. }
$$

The infinite sums in $f_{1}(\xi), f_{2}(\xi), g_{1}(\xi), g_{2}(\xi)$, are well-behaved as $p \rightarrow \pm \infty$, so these can be truncated during computation.

\section{REFERENCES}

[1] S.-I. AmARI, Dynamics of Pattern Formation in Lateral-inhibition Type Neural Fields, Biological Cybernetics, 27 (1977), pp. 77-87.

[2] S.-I. Amari, Heaviside World: Excitation and Self-Organization of Neural Fields, in Neural Fields, S. Coombes, P. beim Graben, R. Potthast, and J. Wright, eds., Springer, 2014, pp. 97-118.

[3] M. Bonilla-Quintana, K. C. Wedgwood, R. D. O’Dea, and S. Coombes, An analysis of waves underlying grid cell firing in the medial enthorinal cortex, The Journal of Mathematical Neuroscience, 7 (2017), p. 9.

[4] P. C. Bressloff, Spatiotemporal dynamics of continuum neural fields, Journal of Physics A: Mathematical and Theoretical, 45 (2011), p. 033001.

[5] S. Coombes, Dynamics of synaptically coupled integrate-and-fire-or-burst neurons, Physical Review E, 67 (2003), p. 041910.

[6] S. Coombes, Waves, bumps, and patterns in neural field theories, Biological cybernetics, 93 (2005), pp. 91-108.

[7] S. Coombes, P. Beim Graben, R. Potthast, and J. Wright, Neural fields: theory and applications, Springer, 2014.

[8] S. Coombes And M. R. Owen, Evans functions for integral neural field equations with Heaviside firing rate function, SIAM Journal on Applied Dynamical Systems, 34 (2004), pp. 574-600.

[9] A. Destexhe and T. J. Sejnowski, Thalamocortical Assemblies: How Ion Channels, Single Neurons and large-Scale Networks Organize Sleep Oscillations, Oxford University Press, Oxford, 2001. 
[10] J. D. T. Engbers, D. Anderson, R. Tadayonnejad, W. H. Mehaffey, M. L. Molineux, And R. W. TURNER, Distinct roles for $I_{T}$ and $I_{H}$ in controlling the frequency and timing of rebound spike responses, The Journal of Physiology, 589 (2011), pp. 5391-5413.

[11] A. Gökçe, D. Avitabile, And S. Coombes, The dynamics of neural fields on bounded domains: an interface approach for dirichlet boundary conditions, Journal of Mathematical Neuroscience, 7 (2017).

[12] J. Guckenheimer, J. H. Tien, And A. R. Willms, Bursting: The genesis of rhythm in the nervous system, World Scientific, Singapore, 2005, ch. Bifurcations in the fast dynamics of neurons: Implications for bursting.

[13] B. Hille, Ionic Channels of Excitable Membranes, 2nd, Sinauer Associates, Sunderland MA, 1992.

[14] R. Hindriks, M. J. van Putten, And G. Deco, Intra-cortical propagation of EEG alpha oscillations, NeuroImage, 103 (2014), pp. 444-453.

[15] M. A. Huertas, J. R. Groff, And G. D. Smith, Feedback inhibition and throughput properties of an integrate-and-fire-or-burst network model of retinogeniculate transmission, Journal of Computational Neuroscience, 19 (2005), pp. 147-180.

[16] J. Huguenard And D. A. MCCormick, Electrophysiology of the Neuron: A Companion to Shepherd'sNeurobiology, Oxford University Press, 1994.

[17] J. R. Huguenard And D. A. MCCoRmick, Thalamic synchrony and dynamic regulation of global forebrain oscillations, Trends in Neurosciences, 30 (2007), pp. 350-356.

[18] C. K. R. T. Jones, Dynamical Systems, vol. 1609 of Lecture Notes in Mathematics, vol 1609. Springer Berlin, Heidelberg, Springer, Berlin, Heidelberg, 1995, ch. Geometric singular perturbation theory, pp. $44-118$.

[19] J. Laudanski, C. Sumner, And S. Coombes, Calcium window currents, periodic forcing, and chaos: Understanding single neuron response with a discontinuous one-dimensional map, Physical Review E, 82 (2010), p. 011924.

[20] L. Muller And A. Destexhe, Propagating waves in thalamus, cortex and the thalamocortical system: experiments and models, Journal of Physiology-Paris, 106 (2012), pp. 222-238.

[21] L. Muller, A. Reynaud, F. Chavane, And A. Destexhe, The stimulus-evoked population response in visual cortex of awake monkey is a propagating wave, Nature Communications, 5 (2014), p. 3675.

[22] P. C. Müller, Calculation of Lyapunov exponents for dynamic systems with discontinuities, Chaos, Solitons and Fractals, 5 (1995), pp. 1671-1681.

[23] D. J. Pinto And G. B. Ermentrout, Spatially structured activity in synaptically coupled neuronal networks: I Travelling fronts and pulses, SIAM, 62 (2001), pp. 206-225.

[24] J. Rinzel And G. Ermentrout, Methods in Neuronal Modeling: From Ions to Networks, The MIT Press, Cambrideg, Massachusetts, 2nd ed., 1998, ch. Analysis of neural excitability and oscillations, pp. 251-292.

[25] J. Rinzel, D. Terman, X.-J. Wang, and B. Ermentrout, Propagating activity patterns in large-scale inhibitory neuronal networks, Science, 279 (1998), pp. 1351-1355.

[26] S. M. Sherman AND R. W. Guillery, Functional connections of cortical areas: a new view from the thalamus, MIT Press, 2013.

[27] G. D. Smith, C. L. Cox, S. M. Sherman, And J. Rinzel, Fourier analysis of sinusoidally driven thalamocortical relay neurons and a minimal integrate-and-fire-or-burst model, Journal of neurophysiology, 83 (2000), pp. 588-610.

[28] M. Steriade, E. G. Jones, and R. R. Línas, Thalamic Oscillations and Signalling, Wiley, New York, 1990.

[29] X.-J. WANG, Multiple dynamical modes of thalamic relay neurons: rhythmic bursting and intermittent phase-locking, Neuroscience, 59 (1994), pp. 21-31.

[30] M. Wechselberger, Geometric Singular Perturbation Theory Beyond the Standard Form, vol. 6 of Frontiers in Applied Dynamical Systems: Reviews and Tutorials, Springer Nature, Switzerland, 2020.

[31] A. C. Yew, D. Terman, and G. B. Ermentrout, Propagating activity patterns in thalamic neuronal networks, SIAM Journal on Applied Mathematics, 61 (2001), pp. 1578-1604. 